\title{
Hair Transplantation
}

\author{
Sreedhar Reddy Pothula and B. S. Jayanth
}

\subsection{Introduction}

In the past few decades, maxillofacial surgeons have played a pivotal role in enhancing the aesthetic value of the face through many surgical and non-surgical procedures, with hair transplantation being one of the popular choices which has seen an exponential demand in the last decade. Though hair restoration procedures were being performed for many decades ever since Dr. Norman Orentreich in 1959 published them in the annals of the New York Academy of Sciences, there has been many advances to the originally described punch grafting which has become obsolete and has been replaced by the so-called micrografts via the two basic techniques of follicular unit transplantation (FUT) and follicular unit extraction (FUE). The success of the above techniques is in part attributed to the theory of donor dominance which postulates that the hair taken from the donor area of the posterior scalp will continue to grow without getting hit by factors which were responsible for the thinning out of hair in the frontal areas [1].

Apart from hair transplant being a promising procedure for male pattern and female pattern alopecia, it has been applied to other causes of hair loss too; traumatic and burn scars on hair bearing areas, alopecia following surgical procedures (i.e. Craniotomy, rhytidectomy procedures), redo hair transplantation, congenital reasons like cleft lip scenario. Currently the scope for hair transplantation is expanding with the demand from patients who want to strengthen a

Electronic Supplementary Material The online version of this chapter (https://doi.org/10.1007/978-981-15-1346-6_34) contains supplementary material, which is available to authorized users.

S. R. Pothula $(\bowtie)$

Oral and Maxillofacial Surgery, Pioneer Advanced Hair Transplant

Centre, Bengaluru, Karnataka, India

B. S. Jayanth $(\varangle)$

ABMSS, Bengaluru, Karnataka, India weak hairline, desire to lower the hairline or fix missing facial hair on the moustache, beard, eyebrow or eyelashes.

As the field of hair transplantation continues to evolve at a breakneck speed with specialists from multiple specialities performing this scientific artistry, it's all the more pertinent that we as maxillofacial surgeons are abreast of the current principles and practice of this hair restorative surgery.

\subsection{Surgical Anatomy}

An understanding of the surgical anatomy of the scalp is necessary to avoid complications and to optimise your performance for hair transplantation.

Scalp consists of five distinct layers with the most superficial layer being the skin which comprises of epidermal and dermal layers. These layers contain hair follicles, sebaceous and sweat glands with rich vascular supply. The second layer of scalp is made up of connective tissue, which is well vascularised and heavily innervated by sensory nerves. The deepest portion of the hair follicle, bulb and papilla, may extend through the skin into this layer (Fig. 34.1a). Thus, when obtaining donor grafts, effort should be made to incorporate the upper part of dermal layer to obtain the entire hair follicle. The third layer also called galea aponeurotica is a tendinous tissue connecting the two bellies of the occipitalis and frontal muscles. Loose areolar connective tissue is the next layer which contains numerous potential spaces, capable of great distension thus allowing for exceptional mobility of scalp. Unfortunately, these spaces are also capable of potential space for haematoma collection and spread of infections. The deepest layer of the scalp defines the pericranium, which is analogous to periosteum throughout the body. 


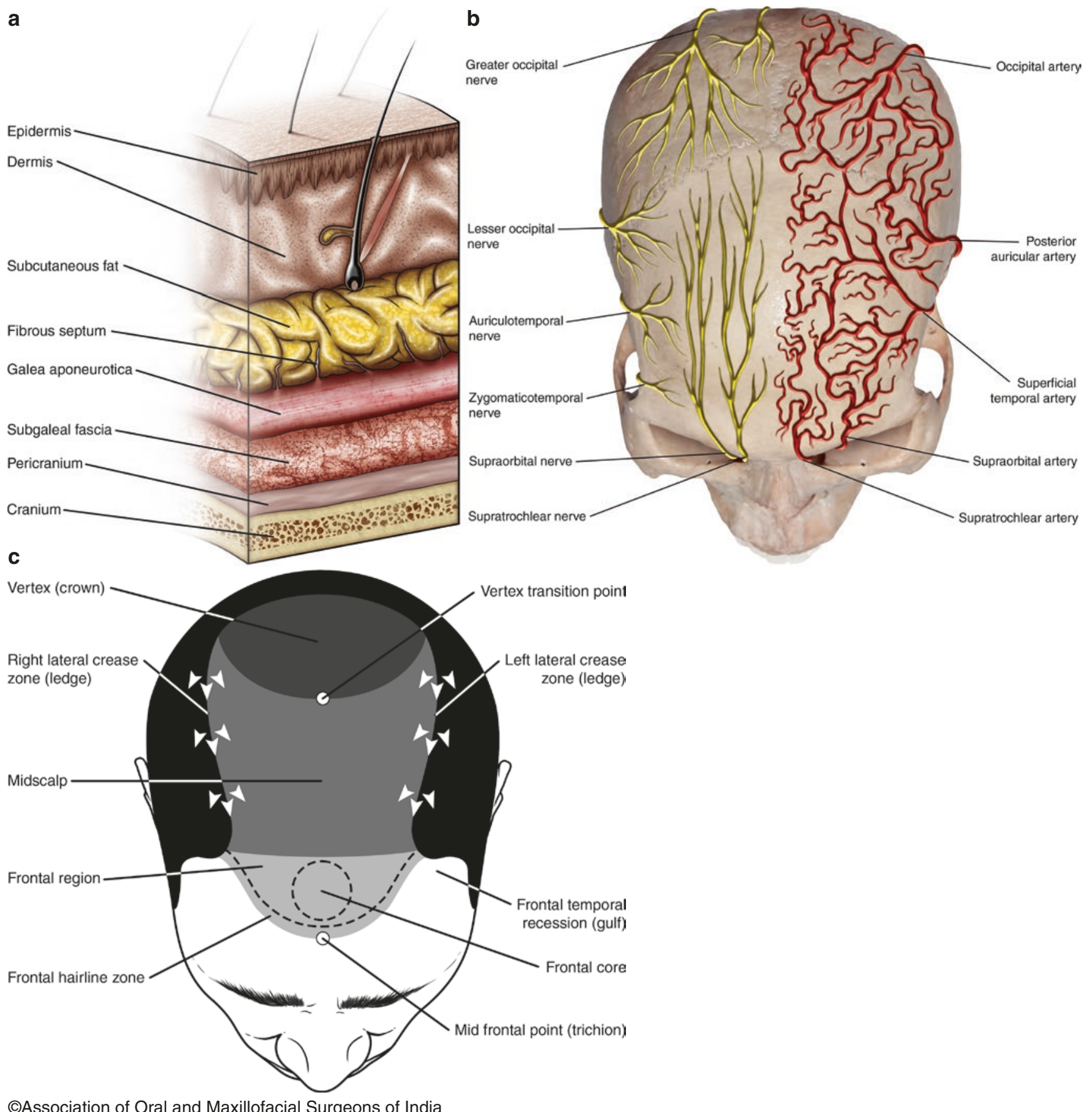

CAssociation of Oral and Maxillofacial Surgeons of India

Fig. 34.1 (a) Hair follicle extending into dermis; note the layers of the scalp. (b) Arteriovenous supply of the scalp with sensory innervation. (c) Zones of the scalp

\subsubsection{Blood Supply and Sensory Innervation}

The arterial supply to the scalp arrives from branches of both external and internal carotid arteries. Branches of the external carotid artery in order from posterior to the anterior include the occipital, posterior auricular and superficial temporal arteries. The supratrochlear and supraorbital arteries are branches of the internal carotid artery. The veins of the scalp follow the same basic network of the arteries and drain into the jugular veins (Fig. 34.1b). All of these vessels freely anastomose in the connective tissue layer of the scalp. Awareness and approximate location of the main branches are very important during the hair transplant surgery not only to avoid haemorrhage or haematoma but also to prevent isch- 
emia to the follicles. Occipital artery which enters the scalp immediately above the external occipital protuberance at the superior nuchal line can easily be injured if depth control is not maintained. If arterial haemorrhage is encountered, electro cautery and suture ligation are adequate to secure homeostasis. Damage to vessels could likely lead to the formation of donor area scar.

Sensory innervation of the scalp arises from all three branches of trigeminal nerve antero-laterally with the forehead getting its supply from the supraorbital and supratrochlear branches of the ophthalmic division, while the anterior temporal scalp is supplied by the maxillary division and the mandibular division goes onto supply the temporoparietal zone of the scalp through its auriculotemporal nerve. The spinal cutaneous nerves of the cervical plexus (c2 and c3) come to supply the area posterior to the auricle via the greater and lesser occipital nerves. These nerves are found in the connective tissue just superior to the galea.

\subsubsection{Zones of the Scalp}

Typically, the areas of male pattern hair loss are horseshoe shaped and are divided into three zones [2] (Fig. 34.1c)

1. Frontal-extends from the hairline to the mid-scalp and is curvilinear. Has a frontal hairline zone anteriorly with the line being irregular like coastline of the sea with mounds and recessions. The follicles in this zone is mainly single hair follicle for the first two to three rows generally blending into multiple follicular units posteriorly giving rise to the density effect known as shingling.

The frontal core zone is a small area in the central aspect of the frontal region which is circular to oval and is very important in framing the face receiving the highest density of grafted hairs.

2. The mid-scalp - is at the top of the head. This horizontal area is bordered on both the sides by the temporal parietal fringes and is sandwiched between the frontal zone anteriorly and the vertex posteriorly.

3. Vertex or crown - the most posterior area being rounded and characterised by whorl arrangement of hair is the toughest area to transplant.

The temporal fringe is the lateral hair zone present on the sides of the scalp anterior to the tragus of the ear and posterior to this is the parietal fringe.

The frontotemporal apex is the area usually devoid of hair at the junction of the lateral frontal hairline and temporal fringe. This coincides with the deepest point of the recession on the forehead and is aligned with the lateral canthus of the eye.

\subsubsection{Microanatomy of Hair}

Hair consists of a living part under the skin extending into the dermis known as follicle and a non-living keratinised part above the skin, the so-called hair shaft (Fig. 34.2). The shaft has three further layers namely the outer protective cuticle, inner cortex and in certain cases the medulla forms the core, and they mainly help in protection and anchorage of the hair.

The growing structure of the hair is the follicle with the upper part being constant and consists of the top infundibulum at the opening of sebaceous gland and isthmus in the middle where the piloerector muscle comes to insert. The lower part of the follicle known as the bulb is very vital as it's the one involved with regeneration and most of the stem cells are located here. Hence, while implanting holding, the graft above or beside the bulb is vital to ensure sustenance of regenerative potential.

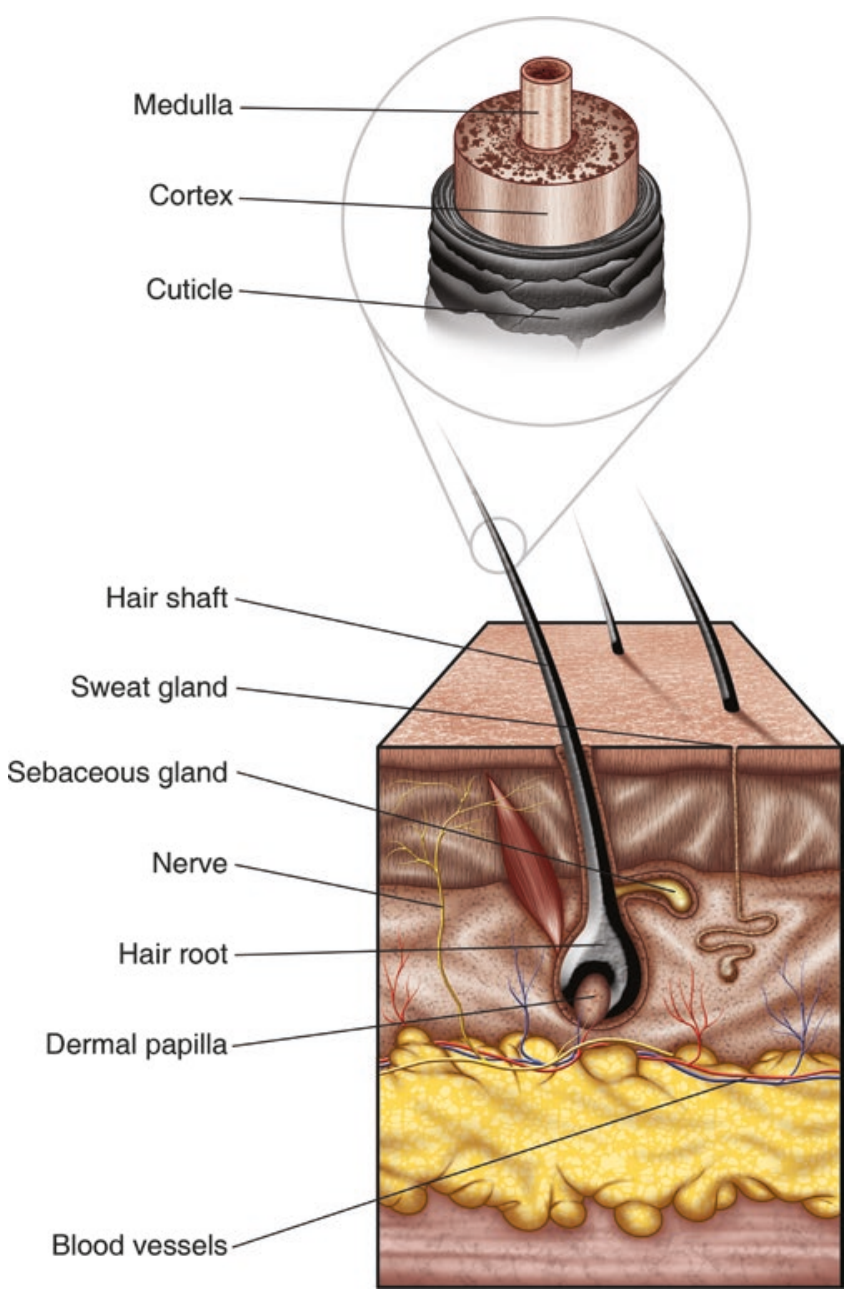

CAssociation of Oral and Maxillofacial Surgeons of India

Fig. 34.2 Microanatomy of the hair 


\subsection{Terminology}

The unique language of hair transplant surgeons consist of a few common words to communicate within the speciality [3] (Box 34.1).

\section{Box 34.1 Hair Transplant Surgery: Terminology}

Follicular unit graft $(F U)$ : The natural Bundle of hair strands consisting of one, two or three hairs woven within a single unit and held by connective tissue elements as the follicle. The graft containing two or three follicular units becomes a multi follicular unit graft.

Micrograft: The follicular unit which has single hair graft within it is referred to as a micrograft, and the same is preferred for frontal hairline implantation.

Minigraft: The follicular unit which has more than a single hair graft and houses four to six hair grafts prior to cutting with microscopes are minigrafts.

Follicular unit density: It's the number of follicular units per $\mathrm{cm}^{2}$.

Hair density: It's the number of hairs per $\mathrm{cm}^{2}$.

FUT: The grafts are obtained from the harvested strip through slivering and dissection of individual follicles under magnification.

FUE: The grafts are obtained individually in the donor area one at a time utilising a punch and handheld extractor.

Stick and place implantation: Refers to implantation made immediately as the recipient site slit is made with a needle or blade.

Pre slit implantation: Refers to implantation made after all the recipient site slits are made with a blade or needle.

Coronal slits: Refers to slits oriented along coronal from side to side.

Sagittal slits: Refers to slits oriented along sagittal plane from front to back.

\subsection{Growth Cycle and Hair Loss}

Hair is cylindrical, outgrowths on the skin made up of keratinous filaments. The normal human scalp contains between 100,000 and 140,000 follicles with hair being arranged in follicular units, small groups of 1-4 hairs each. There are approximately 50,000-60,000 follicular units on the human scalp.

Testosterone is the main regulator of hair growth with the androgens binding to receptors in the dermal papilla influencing the growth. Human hair does not grow constantly and evenly but grows in phases and in cycles which are reproducible. Anagen is the growing phase of hair that accounts for $85 \%$ of the hair growth, during which hair grows at 0.5 inch per month for up to $10 \mathrm{~cm}$ per year with adequate blood supply providing nutrients and minerals to the hair. This phase lasts for anywhere between 3 and 6 years. As long as this phase persists, the hair stays on the scalp and is genetically determined. Anagen is followed by catagen and the later phase onset signals the end of active hair growth. During this transitional phase which lasts for 1-2 weeks, the production of hair stop and hair follicle start to shrink in size and nearly $2-3 \%$ of the hairs are in this phase. Telogen is the final phase of the hair cycle where the aged hair fall out and new ones push themselves out of the skin. This final phase lasts for nearly 3 months with around hundred hairs being shed per day and nearly $10-15 \%$ of the hairs are in this phase at any point in time (Fig. 34.3).

People often confuse with hair fall to hair loss. Hair fall is common in every individual. Around 10-20\% of total hair will be under shedding process. That hair will grow back in 3 months. On average 80-60 hair strands fall is considered as normal.

Male pattern hair loss or androgenetic alopecia as the word suggests is a result of both androgens and genetic factors which work together causing the clinical effect of baldness [4]. Though there are many reasons for hair loss, androgenetic alopecia is the most common reason and accounts for $95 \%$. Fortunately, it can be treated with hair transplant surgery. The diagnosis of androgenetic alopecia is supported by a family history of hair loss, although a positive history is not always identified. There is a slightly greater incidence of having a positive history on the mother's side but the inheritance of male pattern hair loss can come from either side of the family.

In androgenetic alopecia, there is change of hair from terminal hair to vellus hair. There happens to be a gradual evolution of this pattern, with each subsequent cycle of the new hair being fine and thin leading eventually to various grades of alopecia. It initially starts with the frontal hairline recession and regression, followed by loss of more temporal hair and simultaneous thinning of the vertex hair. Norwood classified approximately 7 different types of male pattern hair loss. Identification of these types is key to an understanding of proper planning of hair transplant surgery.

Testosterone gets converted to dihydrotestosterone (dht) both systemically and in the process of intrafollicular conversion under the influence of the enzyme 5-alpha reductase leading to its binding with androgen receptors of the follicular cells in genetically predisposed men leading to inhibition of protein synthesis by shortening the anagen phase, thereby producing finer hairs with shaft becoming thinner; this stunted hair follicle growth leads to miniaturisation of follicles till they become extinct establishing balding [5]. 


\section{Anagen}

(2-6 years)

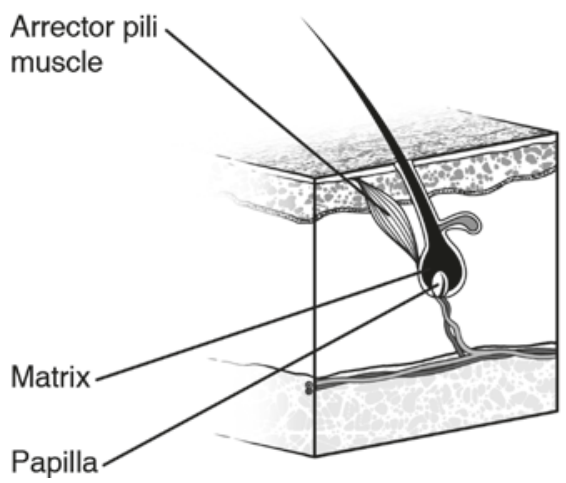

2. Catagen

(1-2 weeks)

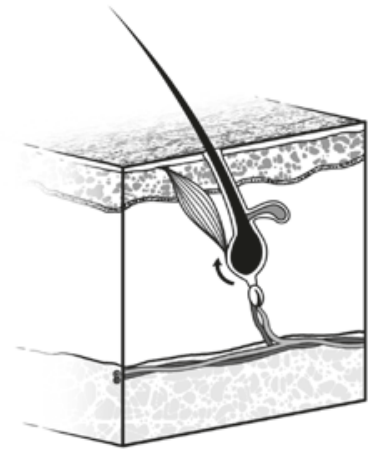

3. Telogen

(5-6 weeks)

CAssociation of Oral and Maxillofacial Surgeons of India

Fig. 34.3 Hair growth cycle

Box 34.2 Basic Requirements for Hair Regrowth

1. Regulating dihydrotestosterone levels in the blood

2. By ensuring normal supply of balanced nutrients to the hair roots

3. Proper blood circulation to carry the nutrients to the roots in the scalp

4. Maintaining good health through general wellbeing of the individual

Clinical studies have given us an understanding that hair can be grown back if the following four things are ensured (Box 34.2):

Hair loss is not a disease but a condition where anagen or the growth phase reduces and the telogen or fall phase is extended. This forms the basis of hair loss in alopecia conditions other than androgenetic alopecia. The factors leading to hair loss could stem from internal/external factors.

Internal Factors There are many factors which internally lead to deficient states harming the hair growth. A few of them which are relevant today are iron deficiency, calcium deficiency, zinc deficiency, general debility, anaemia, hypothyroidism, deficiency of the fat soluble vitamins, thyroid problems, insulin resistance, metabolic disorders like gout and PCOD, prolonged illness, emotional turmoil following surgery and individuals with seborrheic scalp.

External Factors The lifestyle and environmental factors do influence the hair health and growth cycle. The common causes are smoking including passive smoking, stress, alcohol intake, lack of sleep, crash dieting, fad diets, high-protein diet, exposure to extremes of heat or cold, less intake of fluids, poor scalp hygiene, dust, pollution, fumes and exposure to endocrine disrupting chemicals.

\subsection{Classification}

The most popular classification of the male pattern hair loss is the Norwood classification system (1975) which is a refined version of the original Hamilton system (1941) [6].

\subsubsection{Norwood Classification (Fig. 34.4)}

Type 1 presents the normal hairline with minimal recession of the frontotemporal area.

Type 2 patients present a symmetrical and mild recession of the frontotemporal area along with thinning of the hair.

Type 3 patients define balding with minimal or no in the frontal areas and a deep recession.

Type 4 patients present with significant recession and loss of both frontal and vertex hair with a bridge of her between the two still retained.

Type 5 category patients present a progression of the type four category with only thin and narrow bridge of hair between the frontotemporal and the vertex areas.

Type 6 represents the loss of hair that separates the frontal and the posterior vertex areas with further progression in lateral and posterior zones.

Type 7 represents the most severe form of balding with only a band of hair remaining in the low occipital and temporal areas with extensive miniaturisation of the hair.

Basically, in the above classification, the two areas of hair loss - a bitemporal recession and thinning crown gradually enlarge and coalesce until the entire front, top and crown of the scalp are bald. 

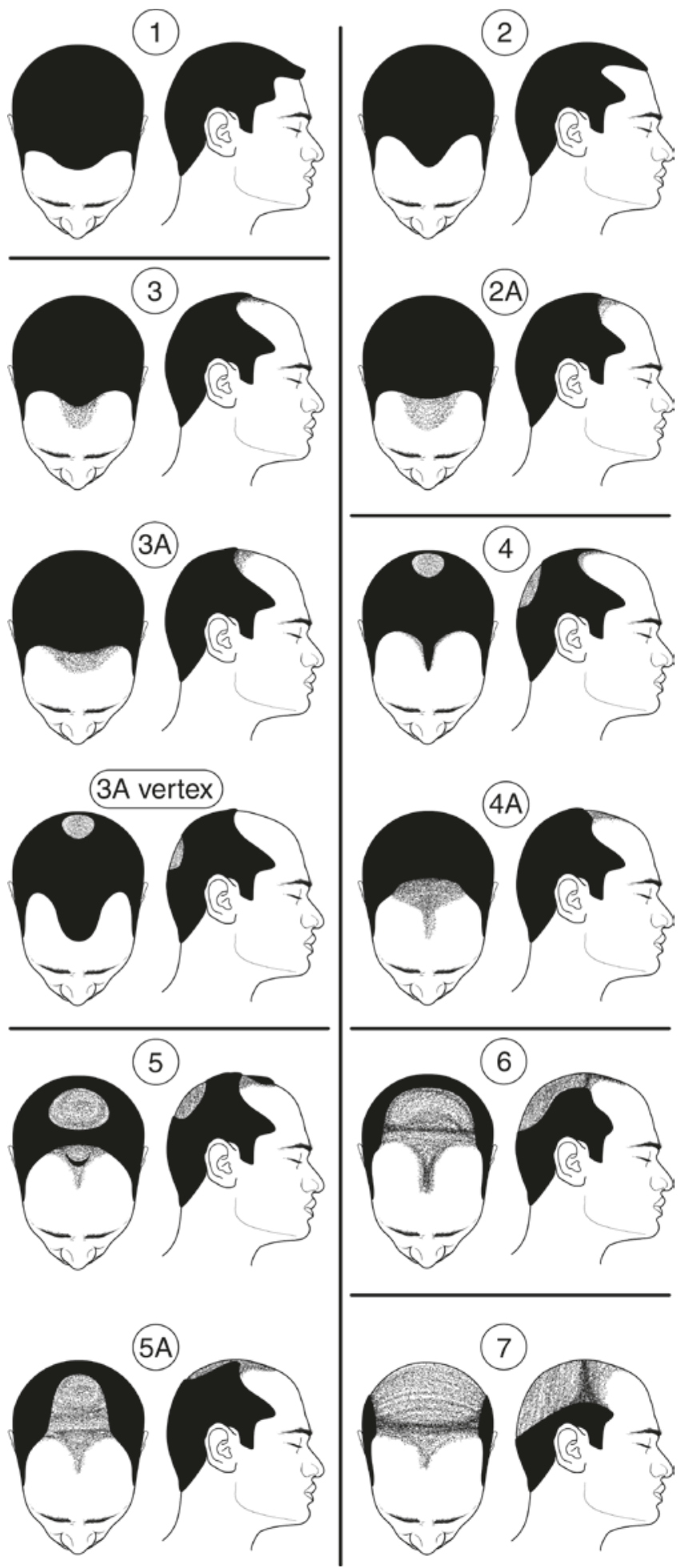

(C)Association of Oral and Maxillofacial Surgeons of India

Fig. 34.4 Norwood classification of male pattern alopecia
To regain the crowning glory, medical treatment gives good results up to type 3 patients while hair transplantation is the only choice for patients between types 4 and 6 .

Routinely, clinicians come across variations of the classification which fall outside the Norwood classification, they are as follows: Differential use pattern alopecia, diffuse and patent alopecia.

\subsubsection{Classification of Hair Loss in Women}

The Ludwig classification (1977) uses three stages to describe female pattern genetic hair loss (Fig. 34.5).

Type I (mild), type II (moderate) and type III (extensive). In all three Ludwig stages, there is hair loss on the front and top of the scalp with relative preservation of the frontal hairline. The back and sides may or may not be involved.

In Ludwig type I, there is early thinning that can be easily camouflaged with proper grooming. Type I patients have too little hair loss to consider surgical hair restoration.

Women with type II hair loss have significant widening of the midline part and noticeably decreased volume. Hair transplantation may be indicated if the donor area in the back and sides of the scalp is stable.

In Ludwig type III, there is a thin, see-through look to the top of the scalp. This is often associated with generalised thinning over the entire scalp. Often patients that have progressed to this stage have too little donor hair to make surgical hair restoration worthwhile.

All women experiencing hair loss should have an accurate diagnosis made, preferably by an experienced dermatologist. This is particularly important since the diffuse hair loss that women typically develop, can occasionally be caused by a number of treatable medical conditions. Regardless of the extent or cause of hair loss, only women with stable hair on the back and sides of the scalp are candidates for hair transplantation.

Other infrequent alopecias observed among the patients visiting hair clinics are as follows:

Alopecia areta: it's an autoimmune disorder which starts with small round and punched out areas with no hair. Lesion has very smooth skin and not even a single hair is present in the lesion.

Alopecia totalis: there is total loss of scalp hair.

Alopecia universalis: there is total hair loss in the body including the eyebrows. 
Fig. 34.5 Ludwig

classification for hair loss in women.
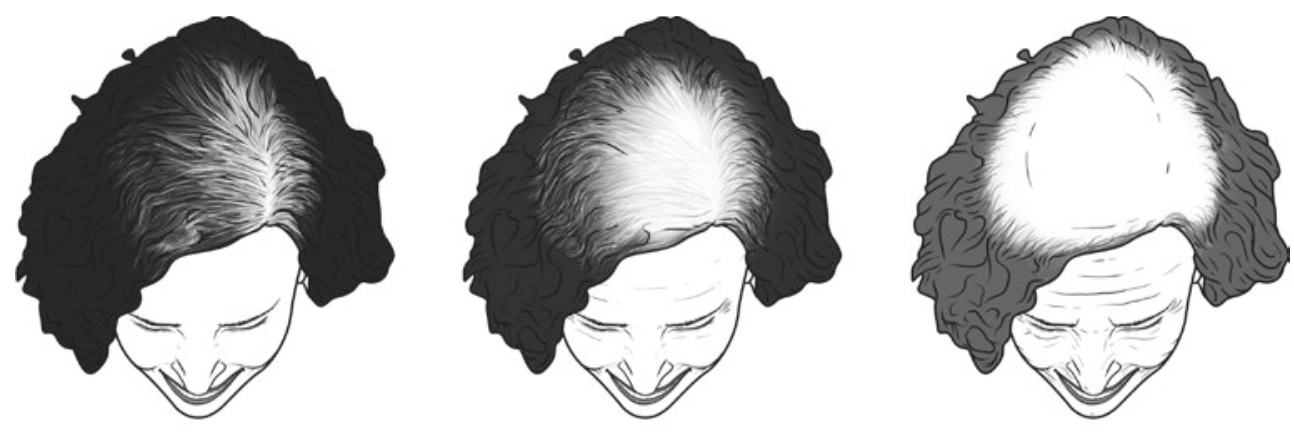

Ludwig scale

CAssociation of Oral and Maxillofacial Surgeons of India
Turban alopecia: caused due to tight turban usage. It affects frontal and parietal areas mainly and is seen among Sikhs.

Trichotillomania: a psychiatric disorder where the individuals deliberately pull their hair every day leading to hair loss in those areas.

\subsection{Medications for Hair Loss}

Only 2 drugs have been approved by FDA and have proven their efficacy in the management of hair loss with good success.

\subsubsection{Minoxidil}

This is a direct hypertrichotic drug, which was initially approved as an antihypertensive agent and was found to have side effect of hypertrichosis. Today, it is available as an over the counter drug with $2 \%$ formulation for women and $5-10 \%$ formulation for men as topical solution and gel. It improves hair count, weight and growth of hair. Post transplantation too it has been found to decrease the shedding of hair and enhanced the time to onset for hair growth. According to few studies, minoxidil acts as a vasodilator by opening up the potassium channels and thereby enhances the vascularity of the hair bulb which in turn promotes hair growth. However, the exact mechanism is unknown. This topical product needs to be applied to the scalp and not the hair and patients need to be informed about the initial increased shedding of weak hair in the first 4-8 weeks. Further, the patients need to be counselled to apply the product twice a day to maximise the efficacy and to wait for a timeframe of at least 4-5 months prior to seeing the results.

\subsubsection{Finasteride}

Finasteride is a drug which is used only in men for reversal of miniaturised hair follicles. This drug is a competitive inhibitor of the enzyme 5-alpha reductase which blocks the conversion of male hormone testosterone to dihydrotestosterone, and thereby works on the pathogenesis of androgenetic alopecia. It is to be taken orally once a day daily at $1 \mathrm{mg}$. Finasteride can cause loss of libido and oligospermia in few individuals. However, this side effect is reversible once the drug is discontinued.

Other medicines like Dutasteride though being a potent inhibitor of 5-alpha reductase is not that popular in the market as it has got adverse effects. Few natural products like saw palmatto can be used in young individuals who refuse to take finasteride.

\subsubsection{Cyclical Therapy}

It entails giving vitamins with calcium supplements on a single day, followed by iron and mineral combination product on one other day of the week and finally amino acid blend; the three regiments are also advocated that their intake be on alternative days as different nutrients and the daily dosing is avoided. The basis of this therapy is that hair requires nutritional support during their anagen growth phase and also for its maintenance; without nutrients, hair cannot get support and the stimulated hair growth to demonstrate disappointing outcomes. This therapy is the result of pioneering work on the role of nutritional supplements in hair growth.

Platelet-rich plasma (PRP) is an autologous biological modifier which has many concentrated growth factors in it and has demonstrated good results in reducing hair loss and has shown to enhance hair counts. It is prepared in a three- 
step process where blood is drawn, processed and injected into the scalp. It is injected into the scalp once every fortnight for 4-6 sittings and this can be combined with the standard therapy.

\subsection{Clinical Consultation}

It is important to begin the consultation by knowing the general health of the patient with a focus on endocrinal conditions affecting hair namely the thyroid disease, any uncontrolled diabetes, polycystic ovarian disease in the case of females and also enquiring specifically about family history of hair loss, past medical management, understanding personal hair care regimen and any past hair transplants done.

The next focus of the clinician during consultations should be on the expectations and motivation levels of the patient trying to drive home the message that it's a progressive condition especially in younger patients who are demanding and want a low hairline. It's important to have a family member during consultation and ensure that they understand that any future hair loss is understood and you are only trying to frame the face. The pattern of the hair loss also needs to be well documented.

The donor site assessment will verify the presence of any white hair, check the elasticity of the scalp, the density of the hair follicles per square centimetre, note the calibre of the hair (thick vs. thin), presence of curly or straight hair, rule out any existing scars and skin pathology including dandruff and infections.

The assessment of the recipient site will focus on the quality and quantity of remaining hair, ascertaining the grade of balding and the pattern of hair loss, previous results of hair transplantation, the skin to hair contrast, direction of the existing hair and existing skin condition. Based on the priorities of the case and having assessed the areas requiring hair transplantation, the number of follicular units required per square centimetre (around $30 / \mathrm{cm}^{2}$ ) is calculated to arrive at the number of follicular units to harvest.

\subsection{Concept of Hair Transplantation}

Hair transplantation as performed today is basically microfollicular unit transplantation and few clinicians call it as hair restoration as there are many methods to achieve the results.

In the early days, hair transplant concept started with flap rotation techniques requiring procedures to be performed under general anaesthesia. These invasive techniques did not gain popularity as the results were not satisfying and the procedures left disfigurement in few individuals. Later on dur- ing the evolution of techniques, macro and micrografts being 5-6 $\mathrm{mm}$ in size were introduced which were harvested with biopsy punches and the resulting grafts were implanted. These plug like grafts being unnatural in appearance gave a classical doll's hair appearance after growth and got phased out with further refinement of hair transplant procedures.

By around 1963, follicular unit transplantation or strip technique was introduced which became a workhorse of hair transplantation until recently and is still considered to be the gold standard method for hair restoration. In the year 2002, Rossman and Bernstein refined the punch method of harvesting the follicles and named it follicular unit extraction which has seen an exponential growth in the recent years and is today the most popular technique of hair transplantation.

The principle behind hair transplantation is quite simple. The hair that grows on the back and sides of the scalp tends to be permanent in most of the individuals. These occipital and parietal hairs are resistant to androgenic alopecia and are used to implant into the frontal or vertex area which will continue to grow as long as occipital hair grow, but in few individuals with advanced grades even these donor hairs can be miniaturised and lost. That's the reason as to why anticipating the future hair loss is most important factor while planning hair transplant procedure.

Hair transplant as a procedure is successful only when hair loss is stabilised, as it will not stop further hair loss. We as surgeons can only relocate and transfer few strong follicles from donor area to recipient area without increasing any new follicle per se. Many young patients who are frustrated by taking medicines over a period of time will seek hair transplant procedure as a remedy, which is an absolutely wrong decision for the clinicians to encourage. It is imperative on the part of the clinician to stabilise any ongoing hair loss before embarking on the hair transplantation, with individuals below the age of 25 years being denied the scope of the procedure.

Hair transplant surgery demands good planning, great surgical skill and good aesthetic sense on the part of the clinician, as hairs grow in specific direction and angulations for the given area of the scalp, and grafts should be placed in a way which should look natural and cover the scalp gaps thus achieving good density.

Most of the patients would like to know if the results are permanent with guaranteed results. Here, it's the astute clinician who should make all efforts to ensure that patient understands the concept of the hair transplant and the future consequences too, rather than simply promising the results.

The basic concept in hair transplant surgery is one of camouflaging technique which makes them look better rather than taking them back to their crowning glory days of their past during their younger days.

Patient should understand that he may still loose hair further and get worse with his genetic predilection to go bald 
and it's a progressive process. Hair transplant will only help him to maintain hairs which are aesthetically acceptable for his age and few individuals may require future sessions too.

\section{Preparation Prior to Hair Transplantation}

Routine blood test consisting of Complete blood count, glycaemic levels, prothrombin time and serology are done for every case. In patients with any underlying medical conditions, further investigations as deemed necessary for the case are done and a medical fitness is obtained from the physician.

All patients are advised to stop topical minoxidil at least a week prior to transplant to avoid bleeding hampering the progress of the case.

Photographs and consent for the procedure are obtained as a part of standard operating procedure for hair transplantation.

Consenting for the procedure should focus on detailed information of the procedure with clarity given on the results that can be obtained explaining the pros and cons of the procedure, their complications and outcomes. Alternative suggestions should also be given including no surgery, use of camouflage techniques and patients being on only medicines. Adequate time should be given to discuss and understand the proposed procedures. Finally, the proposed treatment plan with the fees proposed should be mentioned in the consent along with written preoperative and post-operative instructions.

The treatment plan formulated needs to account for the quantum of hair to be transplanted, the hairline design, the areas of high priority on the scalp, patient commitment and compliance.

\subsection{Techniques and Description}

\subsubsection{Follicular Unit Transplantation (FUT)} (Box 34.3) (Video 34.1)

FUT is also called strip method or stitch method. It's a gold standard method in hair transplant procedures till date though it's an old method [7]. The modern-day FUT is mainly harvesting a single strip, though earlier few doctors practised with multiple blades to obtain multiple strips which are obsolete now.

Strip method includes taking or harvesting a strip of the skin layer from the donor area usually from the occipital area of the head below the occipital protuberance and above the nape of the neck in the so-called safe zone for harvesting.

Strip measurements are variable with $30 \mathrm{~cm}$ being the maximum length and 1-2 cm width depending on skin laxity of the patient. However, the length and width are variable according to planning arrived at during the consultation with the patient as per the indication of the case. At times we have to limit strip width to $1 \mathrm{~cm}$ if the skin laxity is limited. Usually a strip of $30 \mathrm{~cm}$ in length and $1.5 \mathrm{~cm}$ in width will give around 2000-3000 grafts depending on the hair density of the individual.

Box 34.3 FUT

- Linear scar in donor area.

- Healing time is more in donor area.

- Little post-op discomfort in donor area.

- Difficult in tight scalp, with risk of scarring.

- Can’t use in non-scalp hair like beard or body hair.

- Quality of graft is good

- Less time

- No need for shaving entire donor area.

- We can harvest up to 4000 follicular units in a single session.

- Less expensive

- Follicles Transection rate is very less.

\subsubsection{Assessment of the Donor Hair}

Hair transplant outcomes will greatly depend on the donor hair quality and quantity [8]. The important factors that the clinician has to bear in mind are as follows:

1. The number of grafts that are present per square centimetre

Few individuals have thick density being good or best donors (Fig. 34.6a) while a few have sparse density being poor donors (Fig. 34.6b) with the number of grafts per sq $\mathrm{cm}$ varying between 25 and 65 grafts on an average in healthy Indian adults. It further varies from person to person and sometimes from area to area in the same individual. Usually, occipital area has thick density whereas parietal area has less density. Good observation of the grafts in the donor area is most important for the surgeon to achieve good results.

2. Number of follicles present per graft

This is highly variable with 2, 3 or 4 follicles being present in Indian adults whereas a few have only one or two follicles per graft. The presence of reducing follicles per graft is a sign of the progressive nature of the blading process.

3. Donor skin area

Few individuals have large donor area as their scalp size is larger while a few individuals have donor bearing area which is smaller (Fig. 34.7a, b). This entity will decide the number of grafts that is available for harvesting. 

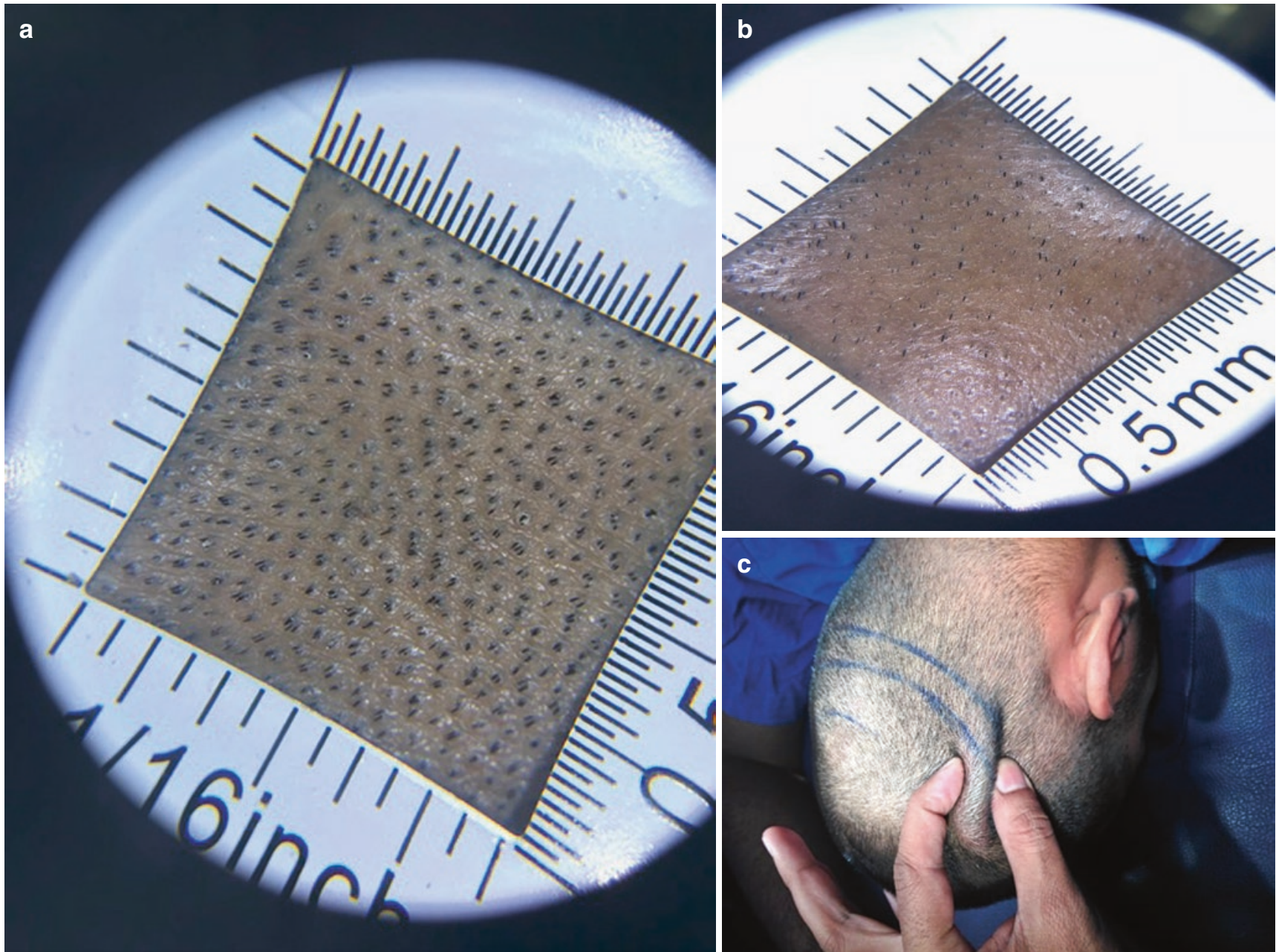

CAssociation of Oral and Maxillofacial Surgeons of India

Fig. 34.6 (a) Good/best donor area based on hair follicular density. (b) Poor donor area based on hair follicular density. (c) Donor area skin laxity testing to ascertain the strip width planned for FUT
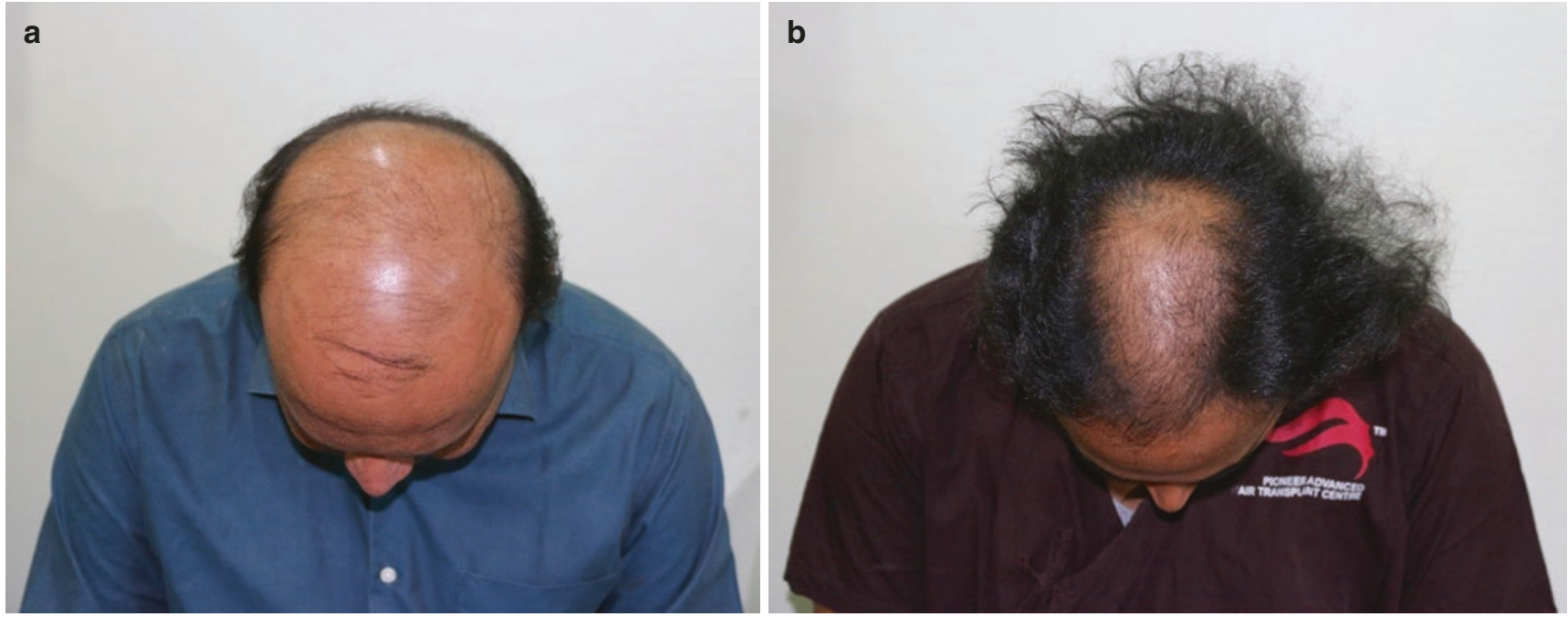

CAssociation of Oral and Maxillofacial Surgeons of India

Fig. 34.7 (a) Large head with more surface area at donor zone. (b) Small head with lesser surface area at donor zone 


\section{Shaft diameter}

Individuals with thick shaft diameter will have better density and the outcome of the results is enhanced.

5. Skin laxity (Fig. 34.6c)

In individuals with better skin laxity, we can harvest more grafts by FUT method by going for wider strips in a single session, whereas the same skin laxity may not have any significance with the FUE method.

Patients who present with straight hair end up optically having less density because light can pass through hair strands and skin becomes visible especially if the hair is black and is contrasting against a fair skin background. Whereas presence of curly and course hair in an individual with dark complexion can give the illusion of thick density.

\subsubsection{Donor Site Preparation and Anaesthesia}

Trimming is essential in the donor site to harvest the strip and the team member in charge of the preparation can ensure trimming the hair to $3 \mathrm{~mm}$ all over the scalp. Few surgeons prefer the procedure without trimming so as to reduce the downtime for the patient's social acceptability. But trimming will ensure better visibility and accessibility to the surgeon while performing the suturing with attention to detail to ensure minimal scarring along the strip harvest. Following the trimming, the next step is to wash the scalp with betadine solution. The clinician should ensure that preoperative photos, consent for the procedure, recording of baseline vitals of the patient and his weight is recorded prior to the preparation of the donor site.

The harvesting of the strip is done with the patient in prone posture following the standards of care with an iv line, monitoring of the vitals with a 3 parameter monitor, and following a dose of prophylactic antibiotic under aseptic conditions. Next, the donor area strip length and width to be harvested are marked out based on the skin laxity tests and depending on the individual's follicular density per square $\mathrm{cm}$.

Once the marking is done, local anaesthesia is administered to the donor area, usually preferring field block in that area. Injecting the local anaesthetic in the lower border of the strip would provide sufficient anaesthesia to the field above.

\subsubsection{Preparation of Local Anaesthesia}

Hair transplant procedure is time taking and lasts around 6-8 h. Lidocaine usually is a very safe anaesthetic with a duration of action lasting between 90 and 120 min and therefore we need to add a long-acting drug-like bupivacaine for hair transplant procedure. Combination of the above would work better for $8-10 \mathrm{~h}$ and the overall dosage should not exceed the maximum permissible as per the weight of the individual. The local anaesthetic solution used is typically as follows:

Lidocaine $2 \% 30 \mathrm{ml}+$ bupivacaine $0.5 \% 4 \mathrm{ml}+\mathrm{ns} 60 \mathrm{ml}$. The above combination gives $64 \mathrm{ml}$ of local anaesthesia, which is of lidocaine $1 \%$.

Tumescence is widely used in hair transplant surgery to raise neurovascular bundles away from the hair follicles which will reduce the trauma to nerves and vessels during the procedure. Another important role for the use of tumescence is in reducing the bleeding during the procedure since scalp bleeds more.

Tumescence solution consists of $500 \mathrm{ml} \mathrm{ns}+1$ ampule of 1:1000 epinephrine and this is injected into the strip area following the local anaesthesia at least $15 \mathrm{~min}$ before the strip harvesting.

\subsubsection{Instruments for the Strip Procedure}

Variscore blade (Fig. 34.8) is useful to have control on the dimensions of the strip and it gives the option to adjust the strip width by adding or reducing the metal spacer plates. Each metal spacer plate comes with thickness of $2 \mathrm{~mm}$ and $1 \mathrm{~mm}$ and, for example, if we need a strip with width of $1.3 \mathrm{~cm}$, then we need to add 6 plates of $2 \mathrm{~mm}$ and 1 plate of $1 \mathrm{~mm}$. The variscore blade has added advantage of depth control while making incision and can accommodate two surgical blades. Usually, for scalp, we can use number 15 surgical blades.

Prior to the incision marking, the strip dimensions are done. Most of the surgeons make straight incision, which may lead to scar. It's always better to follow Langer lines of the skin which ensures less scars with the strip incision looking like a crescent shape with the end tapering off. Strips which are as long as $30 \mathrm{~cm}$ (Fig. 34.9) in length run from above one ear to the other ear. It's always recommended to

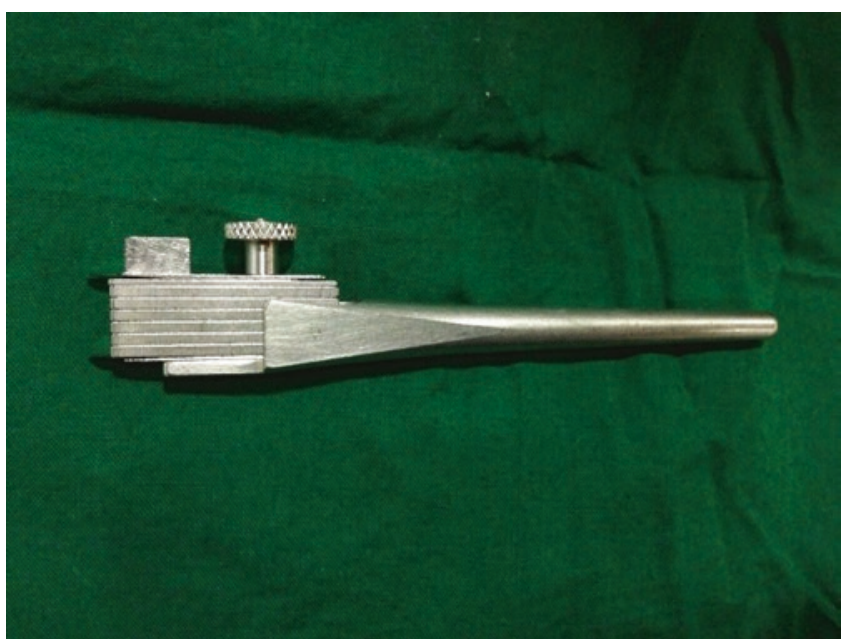

CAssociation of Oral and Maxillofacial Surgeons of India

Fig. 34.8 Variscore blade -popular blade handle for FUT 


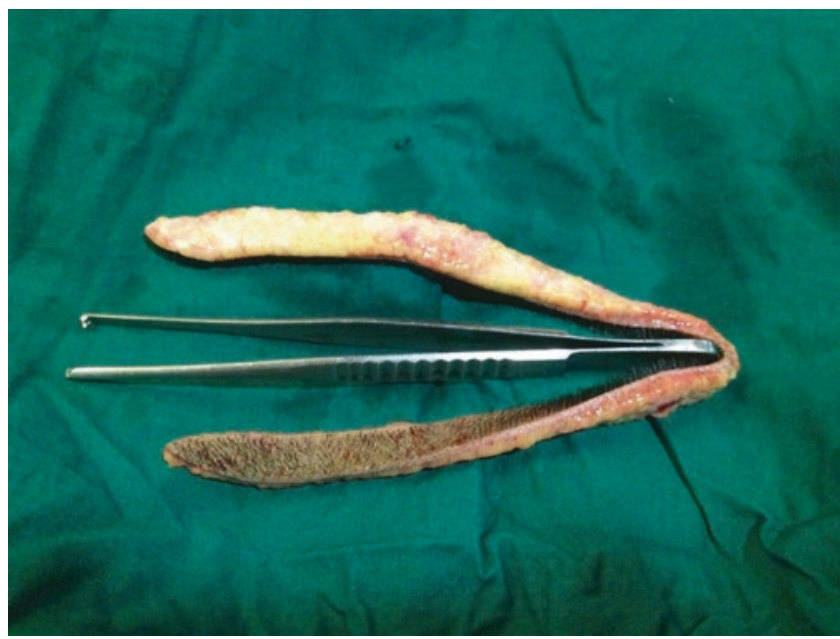

CAssociation of Oral and Maxillofacial Surgeons of India

Fig. 34.9 Strip harvested prior to slivering

place the incision at or just below the external occiput area as the follicles here are considered permanent. It is indeed not good to go too low below the external occiput as we may end up in big blood vessels. After the initial indentation of the incision is done with variscore blade, it is later followed up by the use of BP blade number 15 and the incisions are made very carefully to reach up to $3 \mathrm{~mm}$ beneath the follicular depth with some amount of subcuticular fat around it, while we ensure that we do not cut the galea. While making the incisions, care is taken to prevent slicing of the follicles at the skin margins and the flap is raised from one end to the other using gentle yet deft strokes with the blade.

Good illumination and haemostasis can help to raise strip with minimal damage to both the follicles and vessels in the vicinity. Damage to the vessels is most common if proper tumescence is not attained and can lead to bigger scar and shock loss of hair in the donor area. Any adventure into the galeal layer can lead to haematoma and chances of spreading infections through emissary veins.

Haemostasis is achieved prior to closure by using bipolar diathermy; the scalp wound is irrigated with nor-

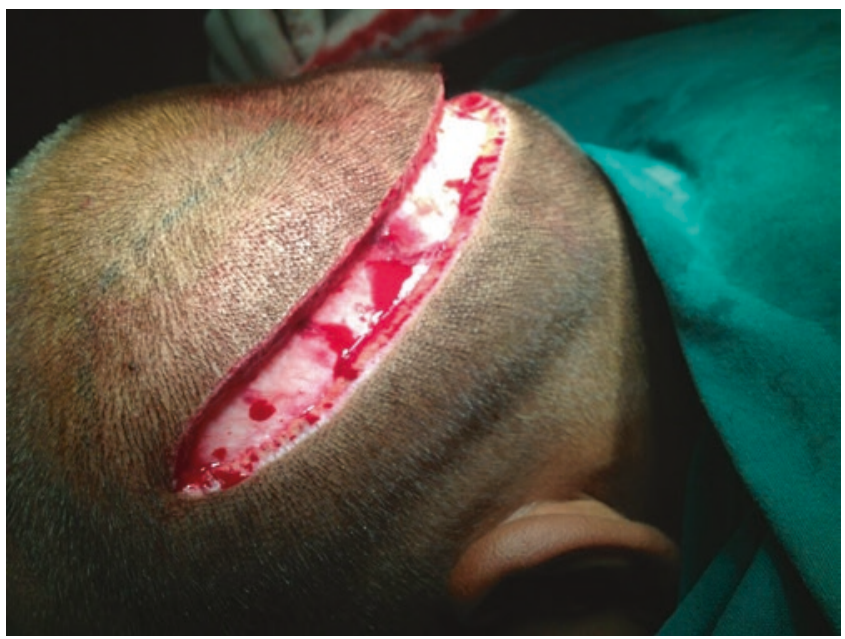

CAssociation of Oral and Maxillofacial Surgeons of India

Fig. 34.10 Wound margins prepared for trichophytic closure

mal saline and a double layer closure is performed with vicryl 2.0 (TS 2404, 3/8 circle, cutting edge) for the inside and a prolene 3.0 (nw800, 3/8 circle cutting edge) for the outer skin (Fig. 34.11a, b). The strip that is harvested is preserved in cold saline on a petri dish at $4{ }^{\circ} \mathrm{C}$.

\subsubsection{Trichophytic Closure}

Trichophytic closure (Fig. 34.10) involves trimming of the upper and lower edges of the skin which facilitates the hair to emerge through the scar ensuring that we will give aesthetically acceptable scar to the patient (Fig. 34.12b) Wound closure done without the above clinical manoeuvre leads to poor scar (Fig. 34.12a), which is quite obvious.

Post-operative care of the wound is important. Antiseptic creams are optional if the donor site wound is washed with clean water or ns on a daily basis. In our experience, suture removal is recommended on 11th post-operative day to avoid wound gaping or wound dehiscence. 
Fig. 34.11 (a, b) Wound closure in layers after Haemostasis
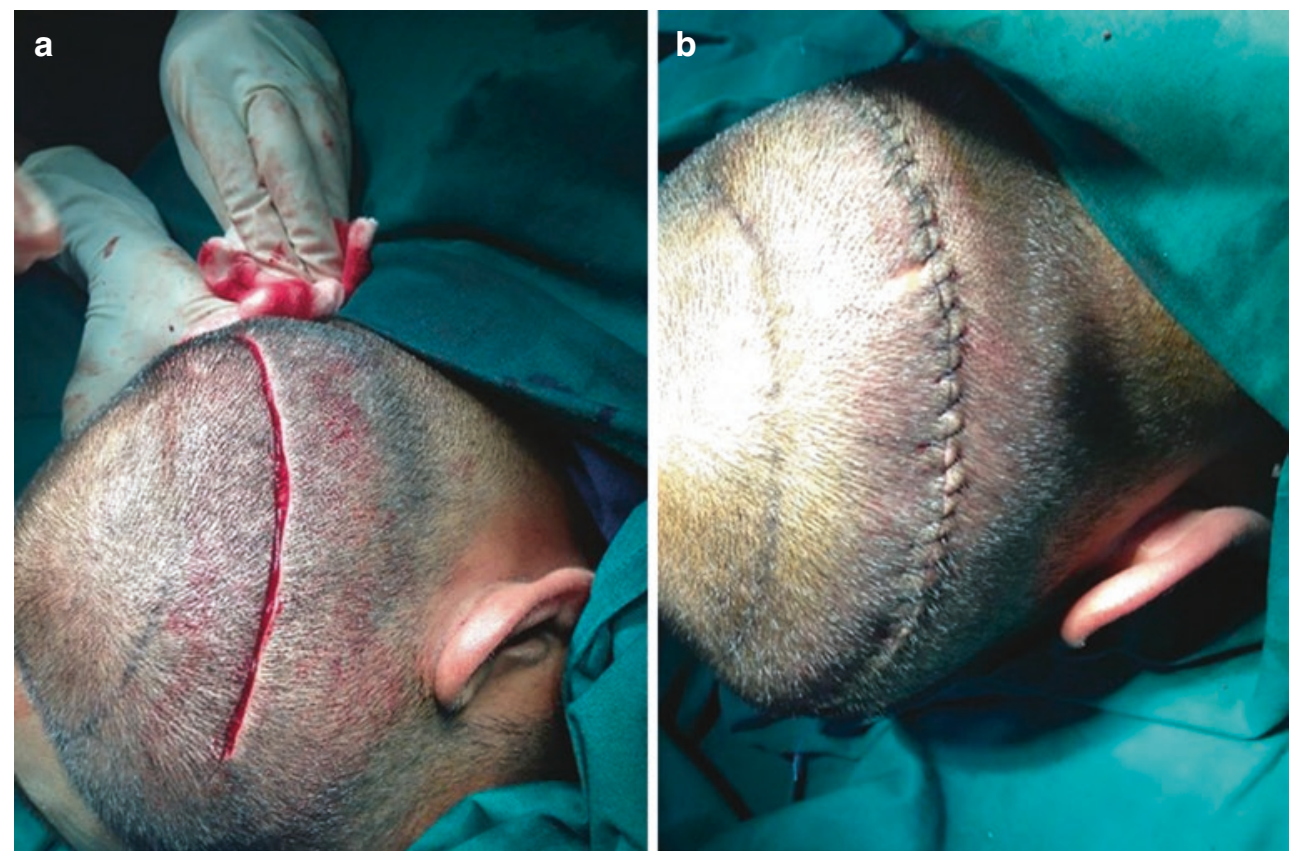

(CAssociation of Oral and Maxillofacial Surgeons of India
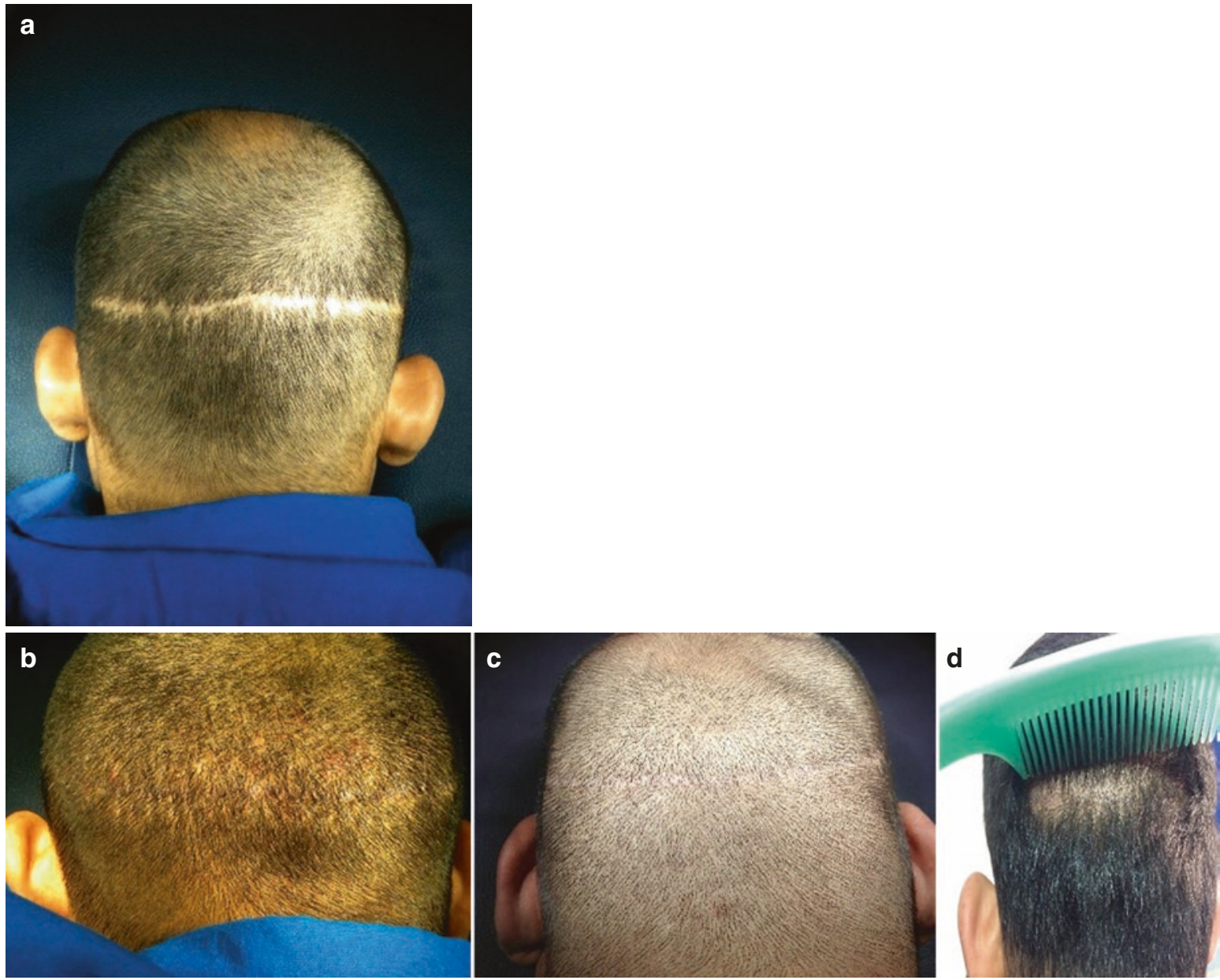

CAssociation of Oral and Maxillofacial Surgeons of India

Fig. 34.12 (a) Poor scar resulting from normal wound closure (b-d) post operative scar following trichophytic closure 


\section{Variable Strip Technique}

This will help us to get maximum grafts with less tension along the scar leading to a better scar. Most of the individuals have thick density in the occipital area and lesser density in the parietal area and any misjudgement will lead to poor wide scar in the parietal areas. Here, by using variable dimensions of the strip technique (Fig. 34.13), we can target for more grafts with least possible scar. In this technique, the strip width is more in the occipital area than compared to the parietal area as illustrated in the image below.

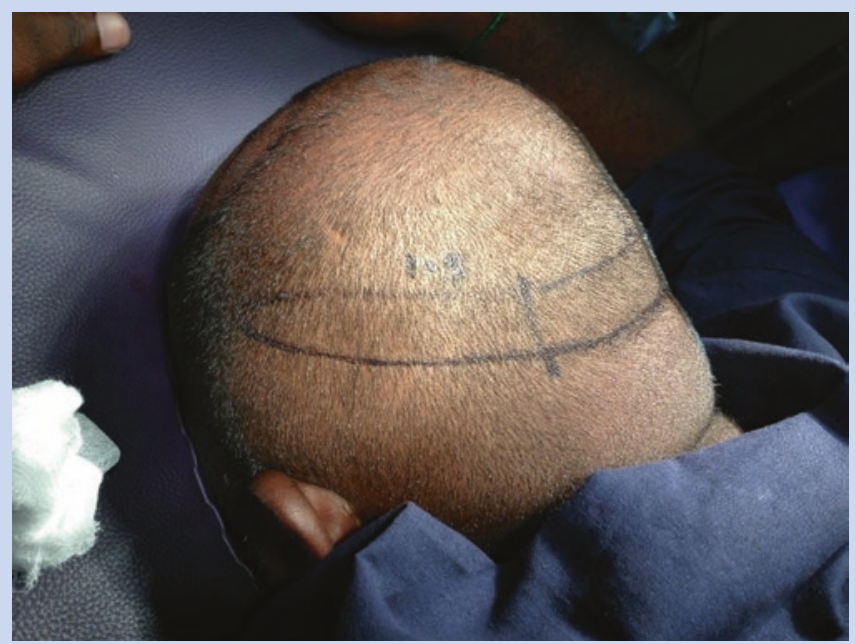

CAssociation of Oral and Maxillofacial Surgeons of India

Fig. 34.13 Variable dimensions of the strip marked out for harvesting

\subsubsection{Slivering}

Slivering is a process where the strip is sliced into small bits of tissues with rows of hair follicles in them, through meticulous deft handwork akin to the baker slicing the bread loaf into thin slices. It is the first step of the separation of grafts from the strip while avoiding any transection of the graft. Higher magnifications should be used to perform this important step for which a few clinics are using stereoscopic microscopes while the others manage with normal 10x magnification. It should be done simultaneously while the surgeon performs the wound suturing to save the crucial graft holding time of around $6 \mathrm{~h}$.

At the end of this step, we get slivers which are ready for the separation process (Fig. 34.14). The separation process is separating individual grafts from the sliver. For the separation process, we need more trained technicians as it is cumbersome exercise and needs great attention to detail to avoid any form of trauma to the graft being obtained with an obvious fight against time.

For the separation process, we need to use $3 \times$ magnification which can be done on a sterilised wooden spatula or an acrylic illuminated sheet.

Wooden spatula would give us good resistance to cut the grafts whereas acrylic sheets will be slippery. Advantage of the acrylic sheet is we can illuminate from below, which is easy for separating the grafts. The separation process is noth-

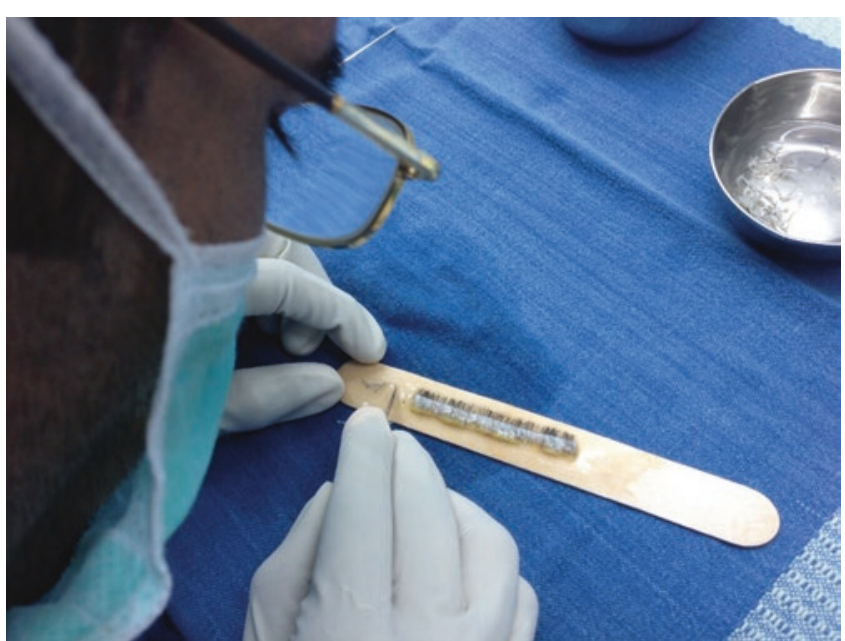

CAssociation of Oral and Maxillofacial Surgeons of India

Fig. 34.14 Slivering and separation of grafts from the strip in FUT

ing but getting rid of the excess fat around the grafts and making the grafts slender in a pear shape or tear drop shape. Care is to be taken to ensure that we do not denude the roots and little fat is left behind for survival of the graft through plasma imbibition. All skin has to be trimmed and removed from the graft and finally these prepared grafts are preserved in cold normal saline around $4-8{ }^{\circ} \mathrm{C}$ temperature. 
While separating the grafts, we would get single, double, triple follicular unit grafts, and care is exercised to maintain the follicular unit (Figs. 34.15 and 34.17a), as it is natural to ensure better survival. These are segregated further in different bowls and are used up for implanting starting with single units for the frontal hairline and progressing onto multiple follicular units as we go posteriorly in a progressive manner.

\subsubsection{Follicular Unit Extraction (FUE)} (Box 34.4) (Video 34.2)

In this technique, we extract the individual follicles one graft at a time with the help of small punches. Also known as no stitch and no cut method, it's minimally invasive procedure
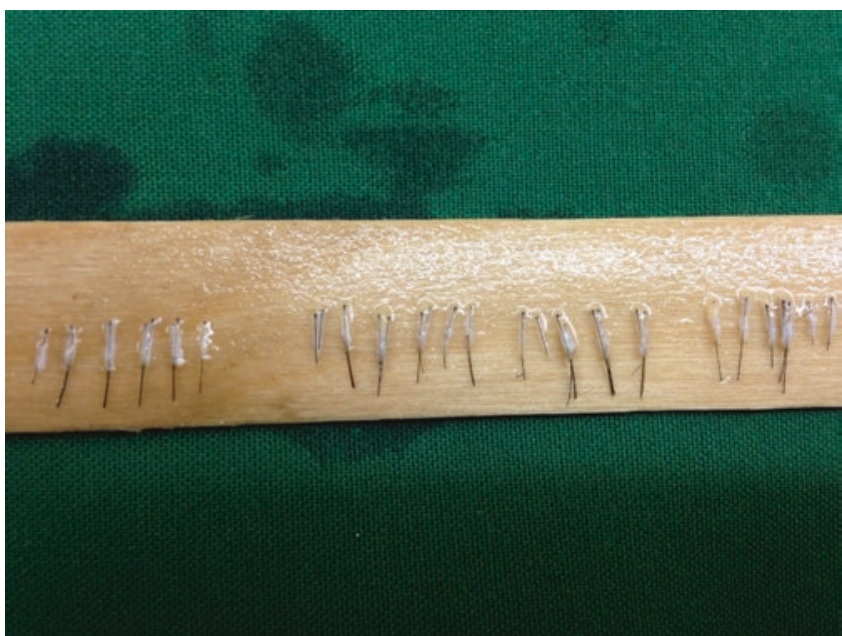

CAssociation of Oral and Maxillofacial Surgeons of India

Fig. 34.15 Separated follicular units compared to the FUT. FUE gained popularity in recent years because of its ease of doing and being less invasive with minimal downtime for the patient. Initially, FUE was introduced with manual punches which was more tedious and time consuming limiting the number of grafts that could be done in a session [9]. Current advances with motorised punch have improved the pace and its effectiveness in harvesting more grafts. Though many different motors and punches are available in the market, normal dental micromotor with straight hand piece would suffice to perform FUE (Fig. 34.16a, b).

Punches are made up of either titanium or stainless steel with the diameter of the punch ranging from 0.6 to $1 \mathrm{~mm}$, with $0.9 \mathrm{~mm}$ being suitable for Indian scalp. The advent of FUE has expended the scope of harvesting follicles from other sites of the body wherein 0.6 or $0.7 \mathrm{~mm}$ punches are useful for beard site and 0.8 or $0.9 \mathrm{~mm}$ punches have been suitable for chest hair harvesting, respectively.

\section{Box 34.4 FUE}

- No linear scar, small round dots in donor area.

- Decreased healing time in donor area.

- No post-op discomfort in donor area.

- Useful for those with risk of donor scarring. Like young pt, very muscular, very tight scalp.

- Useful in Body hair transplant

- Graft quality is not as good as FUT.

- Procedure is time consuming.

- Large sessions required to shave the entire donor area.

- More expensive.

- Follicles transection rate is more

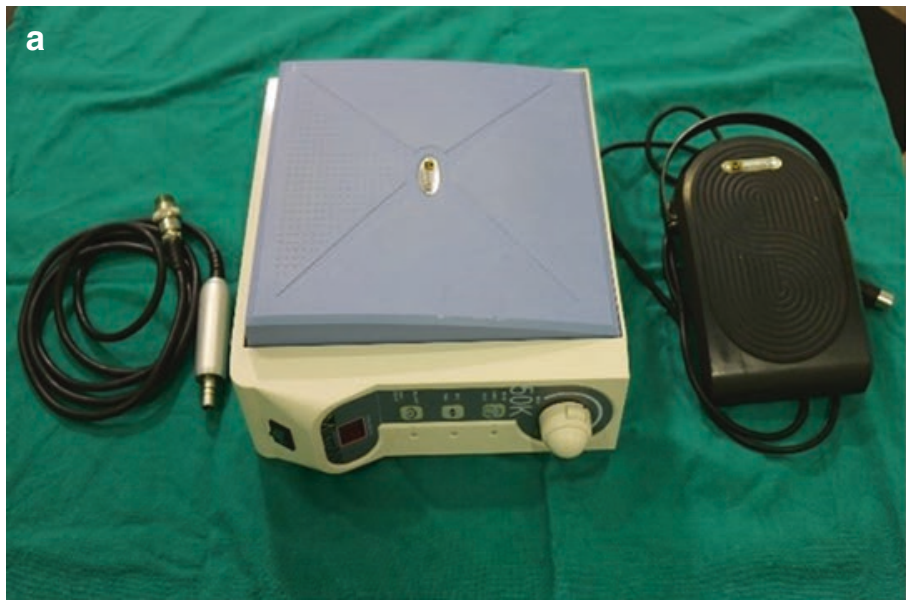

b

CAssociation of Oral and Maxillofacial Surgeons of India

Fig. 34.16 (a, b) FUE instruments: micromotor, straight handpiece and punches 

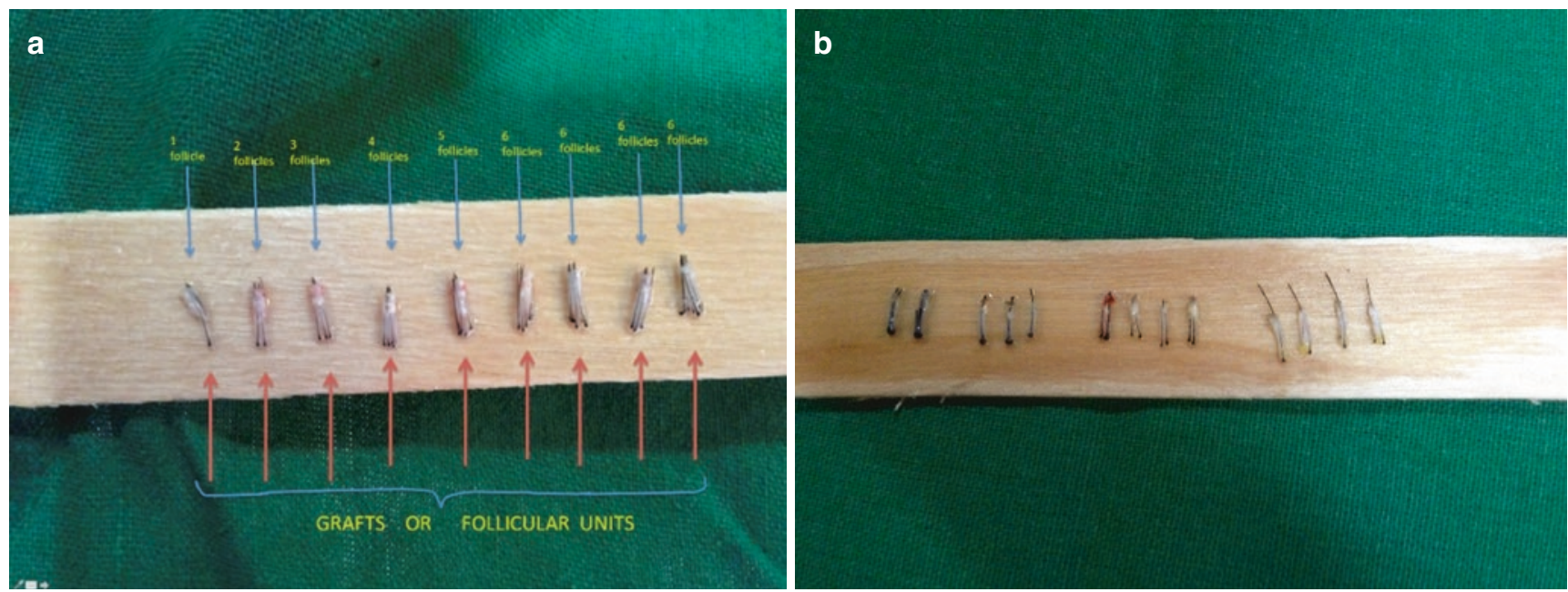

CAssociation of Oral and Maxillofacial Surgeons of India

Fig. 34.17 (a) Follicular units and their natural arrangements. (b) Comparison - FUE grafts on the left which are lean and slender versus FUT grafts on to the right which are bulkier

Punches are available as dull, sharp, serrated and oscillating type. Normal dull or sharp punched would be sufficient to perform FUE. On an average one punch can be used to harvest around 1000-1500 grafts.

This latest motorised technology has made surgeons' job easy and in experienced hands we can harvest around 800 1000 grafts in $1 \mathrm{~h}$, provided patient's skin is favourable.

Being a blind procedure, this is technique sensitive and is associated with a steep learning curve requiring more concentration. It is performed under $3 \times$ magnification controlling the punching around the hair graft while maintaining depth and angulation which is very vital to harvesting the grafts. The punch should get down to epidermis and dermis junction not going beyond $4 \mathrm{~mm}$ from the skin. Surgeon should always analyse the angle of exit of the hair and be parallel it prior to the punching. Acute angles of the grafts would lead to difficulty in punching and lead to not only bigger punch holes but higher transaction rates. Most of the Indian skin is favourable for FUE procedure because of thicker skin types. The follicles obtained by FUE are comparatively slender and devoid of extra adventitious tissue in comparison to the FUT follicles (Fig. 34.17b).

\subsubsection{Blades}

They can vary in size and shape from being thin sharp blade to having a pointed chisel as in the case of sp90 which is one of the popular blades for pre slit technique. Today, the surgeons have a choice of customising the blade with commercially available tools and can use a square or tapered blade as per their choice.

\subsubsection{Needles}

Standard 19 gauge hypodermic needle is preferred for making the graft recipient sites while the same can be achieved with solid core needles eliminating epidermal implantation.

\subsubsection{Limitations of Follicular Unit Extraction}

FUE has an important role to play in hair transplant because it can reach the areas where strip cannot reach to harvest grafts. When we plan second session, FUT may not be possible in unyielding skin types. In poor candidates where donor areas of the scalp have poor hair quality, FUE can be used to target beard and body hairs. FUE like any other procedure is associated with a few disadvantages like comparatively more GTR (graft transection rate), especially in curly hair types and in these few cases graft yield is abysmally low wherein the surgeon will have to convert the case to strip technique. Follicular splay is another situation where hair roots are placed apart which causes difficult extraction. In case of scattered grafts too, the punch may tend to damage more grafts while harvesting and would not be an ideal situation to continue harvesting with high graft transection.

\subsubsection{Hairline (Fig. 34.18a, b)}

The hairline design and location are the most critical factors in the success of hair transplantation, for which one should understand the concept of facial thirds. Till the age of third decade of life, the anterior hairline at the midline is located at a distance equal to the middle third height of the face from the glabella, adding additional $1 \mathrm{~cm}$ in length to it with each passing decade of life to take the hairline upwards and backwards to give a matured look to face considering the progressive nature of hair loss. The frontotemporal apex is the deepest point of the anterior hairline laterally as it merges with the temporal hairline and is located along the lateral canthus of the eye [10].

It is to be borne in mind that the hairline anteriorly is not a straight line but an irregular one having peaks and troughs making it look like a coastline with the front two rows made 


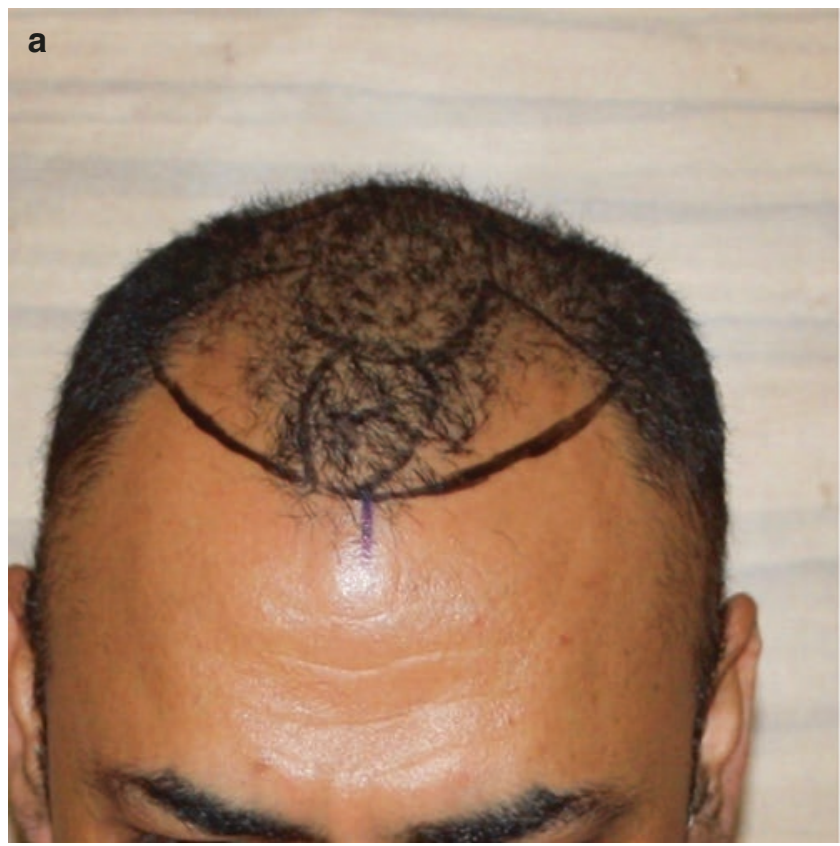

CAssociation of Oral and Maxillofacial Surgeons of India

Fig.34.18 (a, b) Hairline design and natural looks

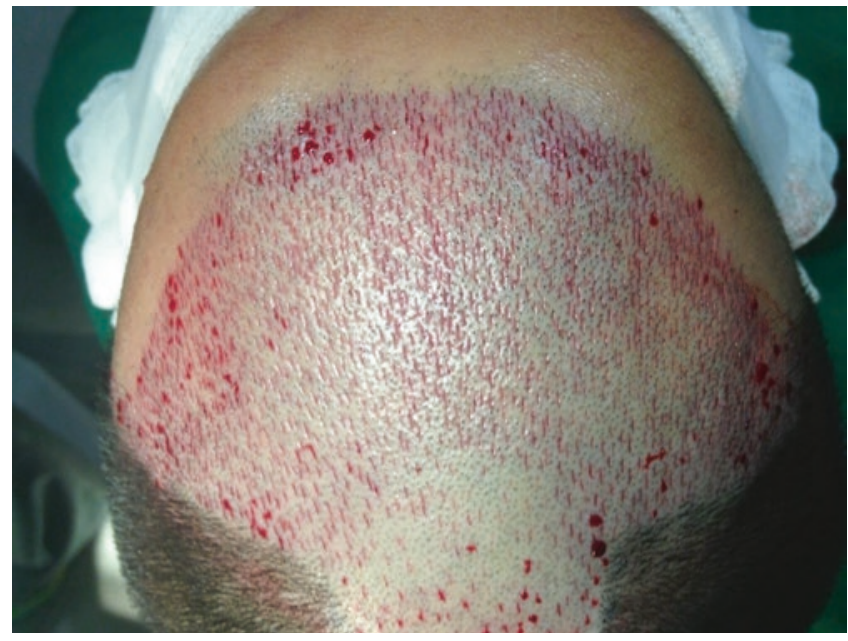

(C)Association of Oral and Maxillofacial Surgeons of India

Fig. 34.19 Recipient site slits

up of single follicular units and gradually it progresses to double and triple follicular units to give the density. The central core or frontal tuft area gets the maximum density of hairs implanted to enhance the strength and aesthetic value of the hairline thus created.

\subsubsection{The Recipient Sites Preparation and Implantation}

Recipient sites are prepared (Fig. 34.19) to receive the grafts by making slits in the skin with either 19 gauge hypodermic needles or sp 90 blades (Fig. 34.20) or other microblades which are of 1-1.1 mm in width. This step could be done
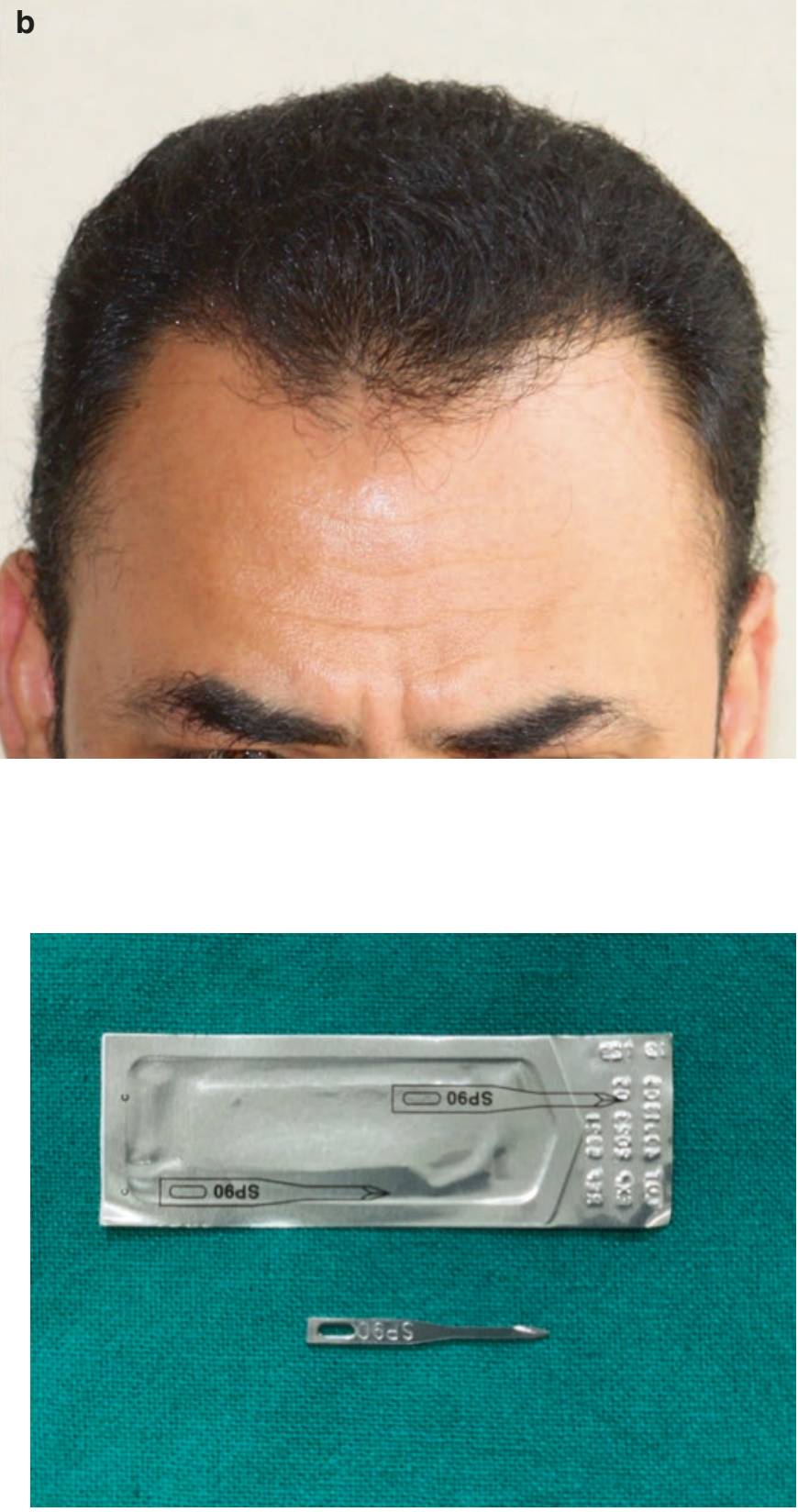

CAssociation of Oral and Maxillofacial Surgeons of India

Fig. 34.20 sp 90 blade used for recipient site preparation

prior to implantation for all the grafts (pre-slit method) or individually for each graft while simultaneously implanting it (stick and place method).

Slits made with blades tend to give more natural looks when compared to needle implantation (Table 34.1). As a prerequisite to facilitate implantation, the site needs to be trimmed short while the remaining hairs guide us in the direction and angulation of the implantation. Proper tumescence will allow us to expand scalp so that we can place more grafts in the given area while also reducing any damage to the neurovascular bundle and enhances visibility. 
The slits are performed in the area with proper direction (converging forwards), depth (follicular length for the given patient) and angulation $\left(15^{\circ}\right.$ in the front $-45^{\circ}$ as we approach the crown part of the scalp).

Once the slits are done, implantation is done by using jeweller's forceps (Fig. 34.21), which are pressure graded. This is also called 2 forceps technique as one forcep is used to locate the slit while the other one is used to implant the graft into the slit. The grafts are to be held above the bulb or beside it where the dermal fat is held.

Implantation of 3000 grafts by forceps would need around 3-3.5 h approximately (Fig. 34.22a).

\subsubsection{Implanters}

Few surgeons use implanters (Fig. 34.22b) which can hold the graft in it and all you have to do is to eject them into the site at the desired direction and angulation. Technicians will preload the graft into the implanter. Implanter can be used

Table 34.1 Advantages and disadvantages of blade versus needle for slit creation

\begin{tabular}{|c|c|c|}
\hline & Blades & Needle \\
\hline Distribution & Uniform & Variable \\
\hline Bleeding & More & Less \\
\hline $\begin{array}{l}\text { Direction and angulation } \\
\text { control }\end{array}$ & Better & $\begin{array}{l}\text { Possible in few trained } \\
\text { hands }\end{array}$ \\
\hline Time taken & Less & More \\
\hline Aesthetic appearance & More likely & Less likely \\
\hline Healing & $\begin{array}{l}\text { Without } \\
\text { marks }\end{array}$ & $\begin{array}{l}\text { Leaves pitting/embossed } \\
\text { scars }\end{array}$ \\
\hline
\end{tabular}

with pre slits or without pre slits made in the scalp. However, implanters tend to create a circular slit which may lead to pitting kind of scars at the base of the graft after growth. They need extra hands for the procedure and come at an extra cost too.

\subsubsection{Combi Technique (Fig. 34.23)}

This is a combination of FUT and FUE in a single session. Combi technique is recommended when we need more grafts as in anything over and above 3500 follicles in a single session. In this technique, the strip is first harvested

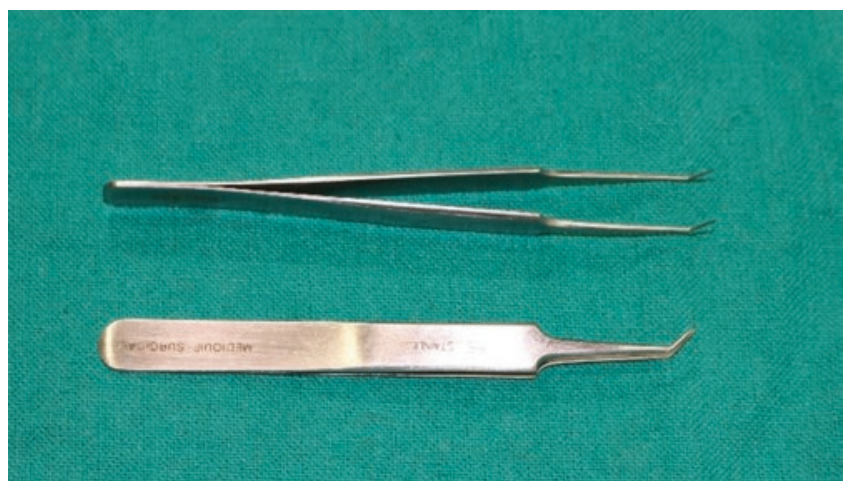

CAssociation of Oral and Maxillofacial Surgeons of India

Fig. 34.21 Jeweller's forceps used for implantation
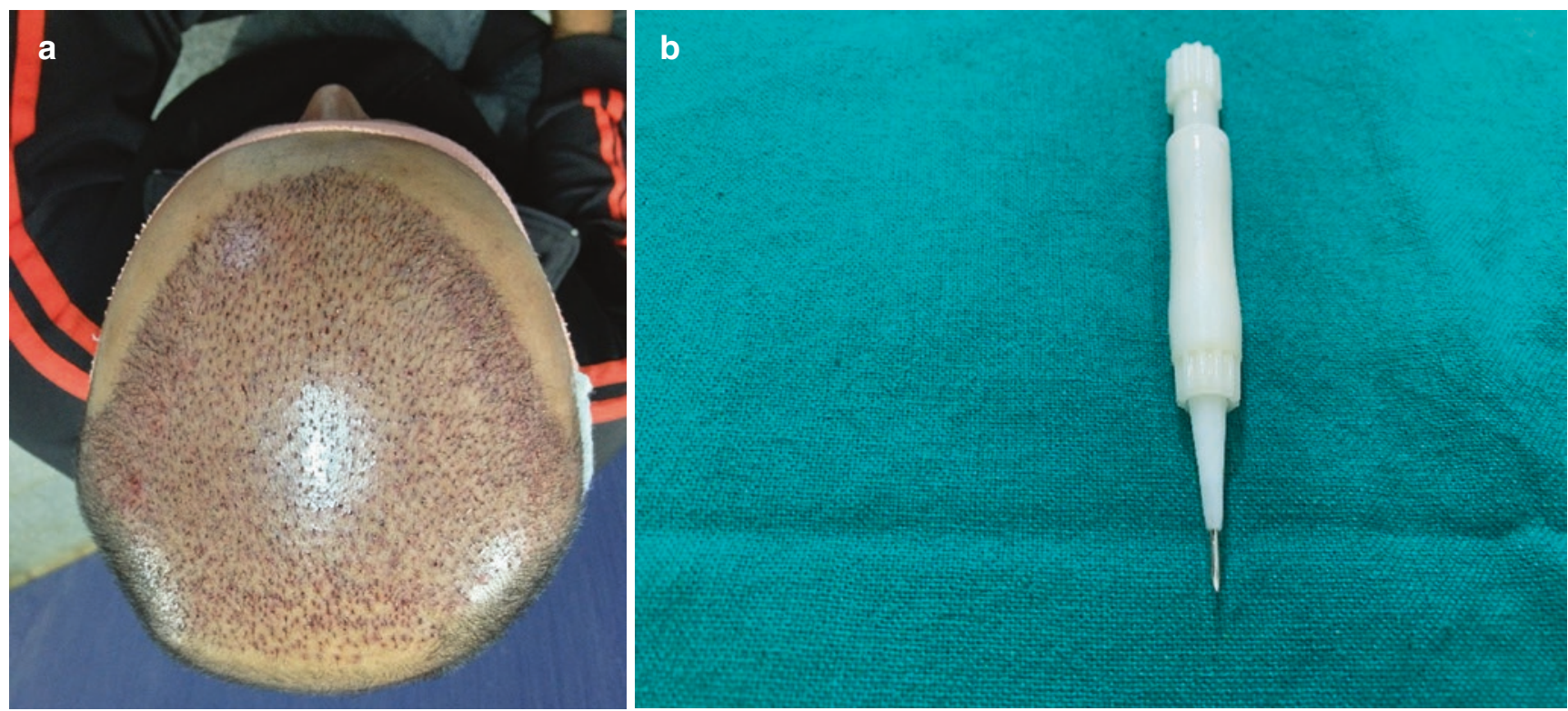

(C)Association of Oral and Maxillofacial Surgeons of India

Fig. 34.22 (a) Immediate view of a case post implantation. (b) Implanters used for implantation 
and then the FUE is performed above and below the strips in the same sitting before implanting. Advantages of this procedure are that the surgeon can reduce the strip length and still can obtain more grafts thereby reducing the strip length and the resulting scar. However, it requires an experienced surgeon and well-trained team to execute the procedure.

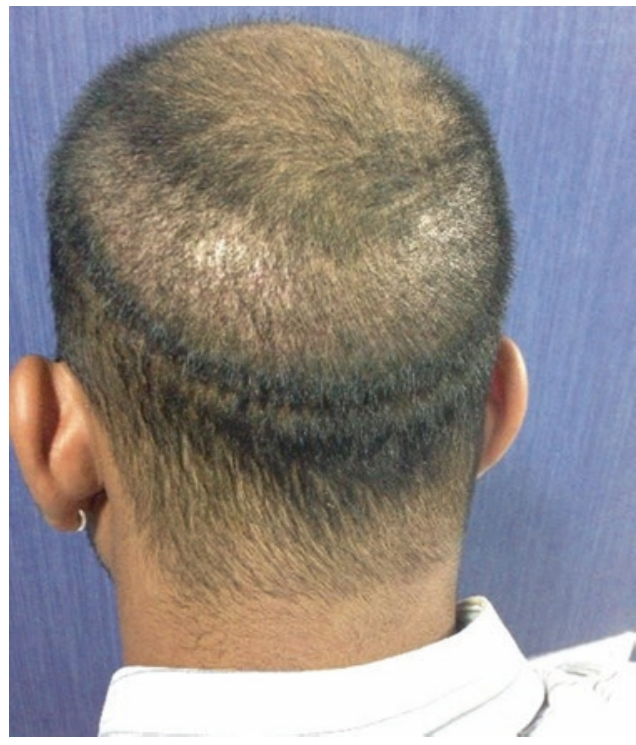

CAssociation of Oral and Maxillofacial Surgeons of India

Fig. 34.23 Combi technique post-operative view

\subsection{Post-operative Care}

Immediately after the procedure, the heaviness, numbness or tightness may be there for a few hours. There is also some swelling on the forehead and temple area due to the tumescence and surgical insult and the resulting swelling is normal. Patients are allowed to sleep on the back or on the sides but are not allowed to sleep on their tummy as otherwise the swelling tends to gravitate onto face and eyes. Patients should be asked to massage the forehead from the middle to the sides for $5 \mathrm{~min}$ every hour for first few days. Rarely some patients may feel momentary dizziness especially while standing up from the lying down position on the procedure table.

There is some oozing of blood-stained fluid from grafted site as well as in donor area (both in case of strip or FUE). Bending forward of the neck can stretch the stitches and can initiate some more oozing from the stitches therefore keeping the chin up is recommended.

Patients are restricted to perform any contact sports/gymming for a period of 12 days and should avoid swimming for up to 3 weeks. They should also not get to field work under the sun for first 3 weeks. Patients who ride a bike are allowed to wear a helmet after a period of 4 weeks. Topical minoxidil can be restarted in the transplanted site after a waiting period of about 15 days post-operatively. Patients are also forbidden from riding a bike or driving in an open vehicle for up to 2 weeks.

After the procedure, antibiotics and analgesics are provided for 5 days. The donor and recipient sites may or may not be bandaged. If bandaging is preferred, a non-adhering betadine pad is placed over the operative sites with several layers of flattened gauze sponges over them to hold the betadine pad in place as the scalp is wrapped with clean bandage using to two to three 4-inch gauze rolls. Crepe bandage can
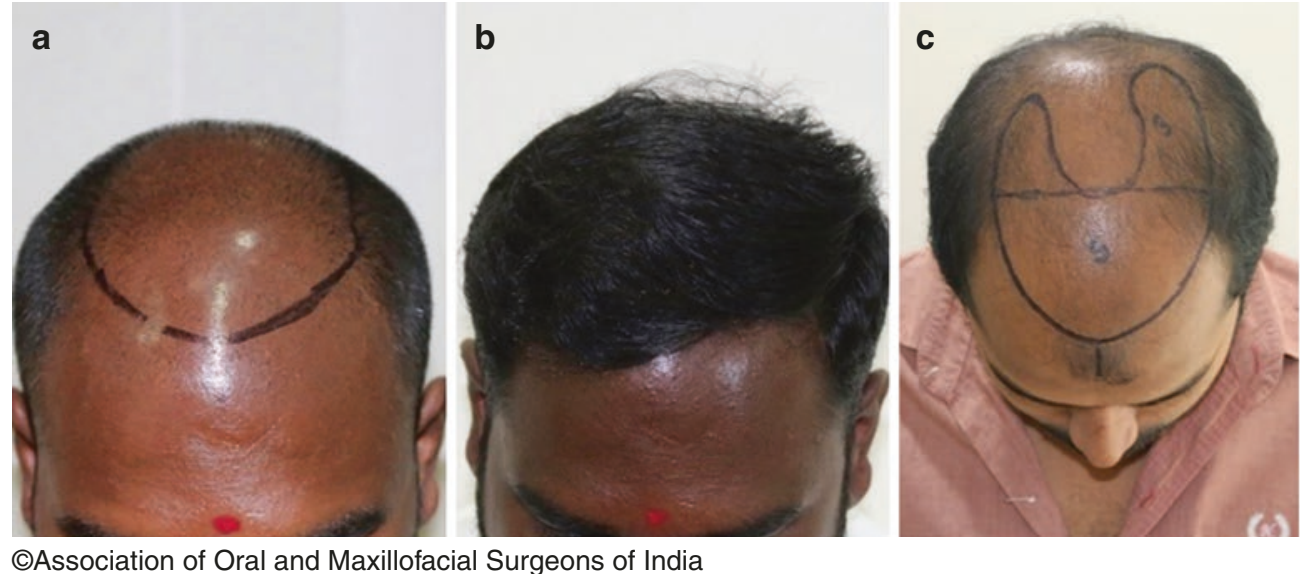

CAssociation of Oral and Maxillofacial Surgeons of India

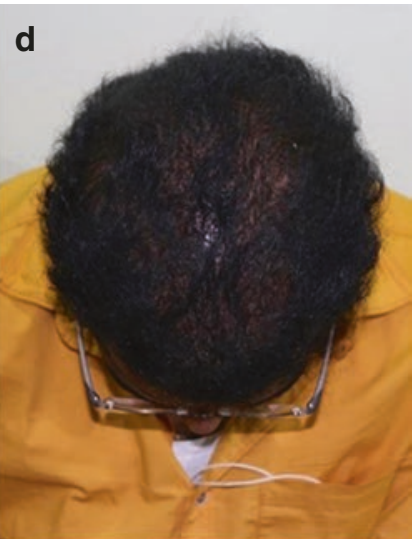

Fig. 34.24 (a-d) Outcome following hair transplantation 
be used over it. The dressing is removed the following morning, and the scalp and each graft are clean meticulously by gently dabbing with a cotton swab with normal saline. Patients are advised to sprinkle or spray normal saline over the grafts every 3-4 h for 8-10 days. Suture removal is performed on 11th day and is not applicable for FUE. Initially for 10 days the grafted area should be washed without rubbing the grafts. Whereas after the above period, grafted area can be washed and patients can rub the grafts. Most of the grafts will be shed by 12-20 days which is a common phenomenon, one need not to worry about it falling off.

Regrowth and Results (Fig. 34.24a-d)

Summary of events following a hair transplant.

Immediate after a hair transplant, there will be mild blood excretion and oozing.

1st day scabs (nothing but dried blood) will form and continue forming for the next few days.

After 4 to 10 days you will notice itching. This is normal, but do not scratch or pick on the transplanted areas.

After 10 days your scalp will be cleared of all scabs.

After 11-20 days your new implanted hair will shed. Now your new follicles are in its dormant (sleeping) stage. It's time to watch and wait.

Pt must be aware that a lag phage exists before hair growth is initiated. After approximately 4 months, the telogen phase to the implanted grafts ends and anagen begins. Complete growth can be seen only after 8 months and hair continue to grow until 12-14 months.

\subsection{Complications}

Able professionals should manage any adverse outcomes by minimising the damage and help achieve the desired outcomes with low morbidity. Fortunately, today the follicular unit hair transplantation is a safe procedure with low rates of complication when performed with care [11].

\subsubsection{Complications in Preoperative Phase}

Overexpectations of the patients are the major cause of complications and arise due to hasty or incomplete consultation and is better addressed with more than one consultation to assimilate the requisite information with the help of informative brochures, websites and discussions with the clinician to arrive at realistic outcomes especially in the younger patients who happen to be unclear and lack maturity for comprehending the results; in such cases, it's better to keep them on medical line of treatment till they become practical in accepting the results. Any individual with body dysmorphic disorder and Norwood vii patients where you would not make much difference would count as poor patient selection by the clinician. In case of ongoing hair loss, the clinicians should not be tempted to promise full coverage or high density influenced by the advent of current medicines as the patients may change their minds and stop the intake of medicines over a long period of time, eventually resulting in inappropriate placement pattern and lack of donor site for harvesting. Clinicians should not be further influenced by the patients' demand for low unaesthetic hairline, or be drawn into performing mega sessions arising out of patients' demand and be careful in choosing the donor material in the middle of the back and sides of the permanent fringe avoiding any slanted scars or scars visible in scanty donor hair bearing areas.

\subsubsection{Complications in Surgical Phase}

1. Pain:

Patients who are counselled appropriately and are provided with a relaxing ambience generally experience minimal discomfort. In-depth local anaesthesia achieved through precise nerve blocks and ring blocks administered with a long-acting drug-like sensorcaine and taking help of vibrating devices while injecting the solution slowly will go a long way in enhancing the comfort levels. Anxiolytics should be prescribed by the clinicians to patients who are apprehensive about the procedure.

2. Bleeding and popping:

Optimising the health of the patient prior to the surgery results in blood pressures being within normal limits. Adequate tumescence consisting of saline and epinephrine has to be injected below the dermis to allow the surgical field to be lifted away from the bleed vessels and then the clinician has started the procedure after 15 min will have minimal bleeding. Too much of tumescent in the recipient site can be a cause of popping, though clinician cannot eliminate popping as pre slit technique can minimise the issue.

3. Bad hair direction:

Clinicians should plan appropriately to maintain the direction and exit angle of the hair that mimics the natural growth. Keeping the grafts with a few millimetres of hair will allow the clinician to ascertain the angle during implantation.

4. Hiccups:

Due to the irritation of the aberrant branch of the vagus nerve, patients can have hiccups through the procedure.

5. Poor graft quality:

A good team leader will constantly supervise to ensure proper handling of the follicles, which will minimise trauma to the follicle resulting from transaction, manipulation, desiccation, oxygen starvation and crushing of the graft [12]. 


\subsubsection{Post Surgical Complications}

1. Donor site effluvium:

Hair in the growth phase can fall rapidly post transplantation due to temporary lack of oxygen during surgery resulting in loss of hair variably along to suture line when significant blood vessels are cut. Any such hair loss will be noticeable around the third week postoperatively and leads to embarrassment to the surgeon. If the donor area can be raised above the blood vessels with good volume of tumescence, the above complication is rarely encountered.

2. Infection:

This is a very rare complication seen today given the standard asepsis precautions followed and is rarely encountered where the patients are not given adequate post-operative instructions especially in cases of tight donor area closure and in patients with compromised immunity.

3. Dehiscence:

A poor surgical technique associated with bad suturing, harvesting wide strips, performing tight closures or resulting infections and necrosis can lead to dehiscence.

4. Dysesthesias:

Today it's no longer a complication thanks to adequate use of tumescence which will lift the hair follicles above the neurovascular bundles.

5. Scars:

This unavoidable outcome can be prevented by following the basic principles of surgery with accurate approximation, closure without tension, by using double layered closure, trichophytic technique and avoiding resolvable suture for closing the skin. Donor scars also occur due to improper donor assessment prior to the surgery. Skin laxity test will help us to evaluate the skin laxity so that we can limit the strip width. At times we may need to consider variable width dimension to reduce the tension in the wound, usually where occipital area skin laxity is more and parietal area skin laxity is less. So we can consider more width at occipital area and less width at parietal area. If skin has less laxity, we can consider FUE.

Any hypertrophic scar can be managed with steroid or injection and later implanting hairs with FUE.

6. Forehead swelling and ecchymosis:

Based on the laxity of the skin in the frontal area and the trauma induced by the slit making process, swelling occurring at 3-4 days post hair transplantation can be mitigated by including some steroids in the la solution, applying pressure bandage and some digital massage across the forehead.

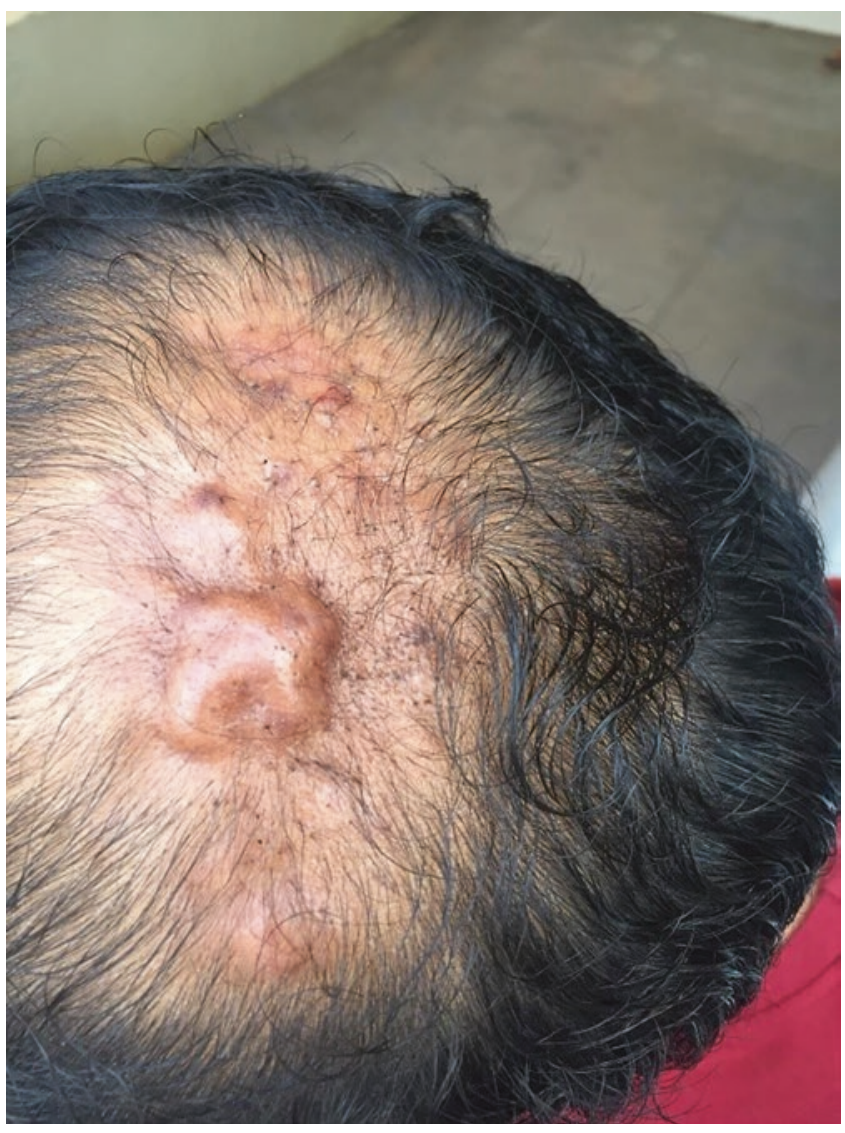

CAssociation of Oral and Maxillofacial Surgeons of India

Fig. 34.25 Folliculitis post hair transplantation

\section{Surgical effluvium:}

This is similar to the donor site effluvium which results in the anagen hairs going into telogen phase and falling out around the second or third post-operative week and regrowing with the grafts. Preoperative use of minoxidil solution tried 1 month prior to the procedure is recommended to prevent the above complication.

8. Cysts, pustules, pimples and folliculitis: (Fig. 34.25)

The above sequelae following hair transplantation can be avoided by preventing deep implantation of grafts, transacting hair during site creation and piggybacking of one follicle up on another. Most of them do settle down with time; however, acne like therapy can be offered to resolve the lesions faster.

9. Pitting and tenting:

It's very important to avoid the above complication in the frontal hairline which could otherwise be unacceptable and are easily preventable by avoiding deep placement of the grafts as in the case of pitting and too superficial placement of the grafts as in the case of tenting. 


\section{Poor growth:}

This can be attributed to poor handling of the grafts resulting in physical and biological trauma to the grafts associated with dehydration of the follicle, crushing of the follicle during implantation, thermal insult and prolonged holding time outside the body.

The incidence of complications may be reduced by avoiding mistakes by the team in general (Box 34.5) and those related to the technique, specifically graft survival (Box 34.6).

\section{Box 34.5 Frequently Made Mistakes Made by Team}

- Too much cross talk between team members annoying the patient.

- Members do not address patient by name.

- Grafts prepared either too chubby or skinny.

- Too many single grafts prepared.

- Photography not taken properly.

- Consent not taken properly.

- Grafts allowed to dry.

- Not interested in routine work.

\section{Box 34.6 Factors Affecting Graft Survival}

- Proper donor tumescence

- Temperature of holding solution

- Dehydration of follicle

- Crush injury of follicle

- Transection of follicle

- Adequate dermis and fat around follicle

- Smoking

- Biological modifiers

\subsection{Current Advances}

Futuristic developments in the field of hair transplant need to focus on the current lacunae in the procedure and revolves around the availability of limited donor supply and the duration of the procedure.

Low-level laser light therapy has been approved by FDA as a device to stimulate the hair growth in the $650-800 \mathrm{~nm}$ spectrum. Home-use scalp brush or helmets with laser technology are available which use the pain-free technology to stimulate epidermal stem cells in the hair follicle to move the follicles into anagen phase.
Automation of the FUE process with the use of robotics has shown to reduce the follicular transaction rates and improve the harvest speed, resolving the issue of skills possessed by practitioners. Artas and neo-graft are devices which have glamorised the field by involving robotic technology, but the running expenses and need to create sites and manually implant follicles need to be solved in the coming days.

If hair can be cultured from the scalp biopsy, then the patient would not need to donate hair by going through a painful procedure, by cloning hair we can create unlimited supply of follicles and avoid ration of hair. Current state-ofthe-art research promises that the technology would get transferred from bench side to the bedside in a few years from now.

\subsection{Role of a Maxillofacial Surgeon}

A maxillofacial surgeon makes a good hair transplant surgeon as they are well trained in surgical anatomy of the head and neck with a detailed knowledge of flap design and through in-depth working knowledge of the local anaesthesia management. They have a great aesthetic sense with artistic hands and are well versed with cephalometric analysis which comes handy in judging facial proportions and hairline placements, further most of them are very well adapted to handling the rotary handpiece from their formative training. Maxillofacial surgeons are well trained exclusively in head and neck surgery for 3 years and are competent at managing wounds and infections, thus justifying their qualifications to perform hair transplantation alongside plastic surgeons and dermatologists.

\subsection{Conclusion}

Surgeons who deal with aesthetic surgeries on the face need to have a good range of skills to deal with all aspects of beauty which are defined by the individual elements. The most important of them all being framing of the face like a photo frame with hair transplantation which will allow us to focus our vision on the finer elements of the facial beauty be it the nose, lips, eyes, teeth, jawline or a good smile. If surgeons follow the principles of hair transplantation surgery, they will create patients with high degree of satisfaction using the micrografts of FUE or FUT procedure. This sophisticated form of art will bring a sense of gratification to oral and maxillofacial surgeons by embracing it as a tool along with all other tools at his disposal. 


\subsection{Case Scenarios}

\section{Case Scenario 1 (Fig. 34.26)}

Figure 34.26: male pattern baldness-Norwood classification 4.

Treatment: FUT method with 2850 plus microfollicular grafts transplanted.

\section{Case Scenario 2 (Fig. 34.27)}

Figure 34.27: male pattern baldness class 5 .

Treatment: 4000 follicular units transplantation done through Combi technique involving both FUT and FUE in the same session.

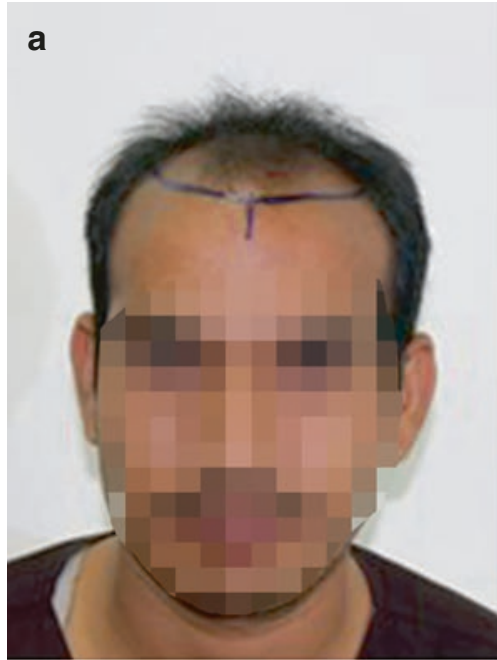

d

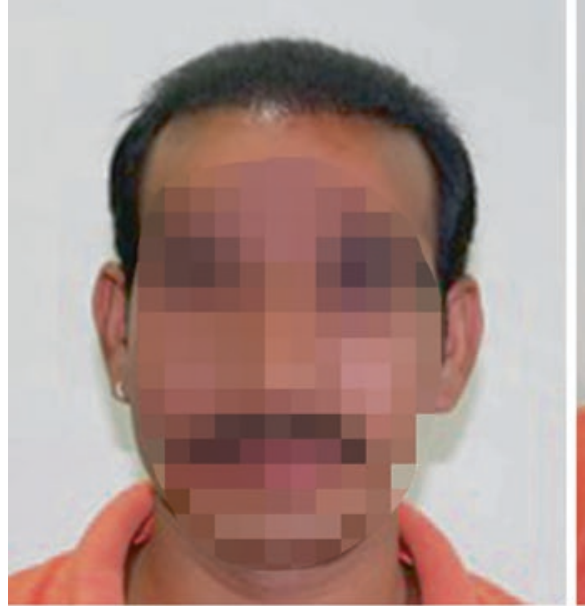

CAssociation of Oral and Maxillofacial Surgeons of India
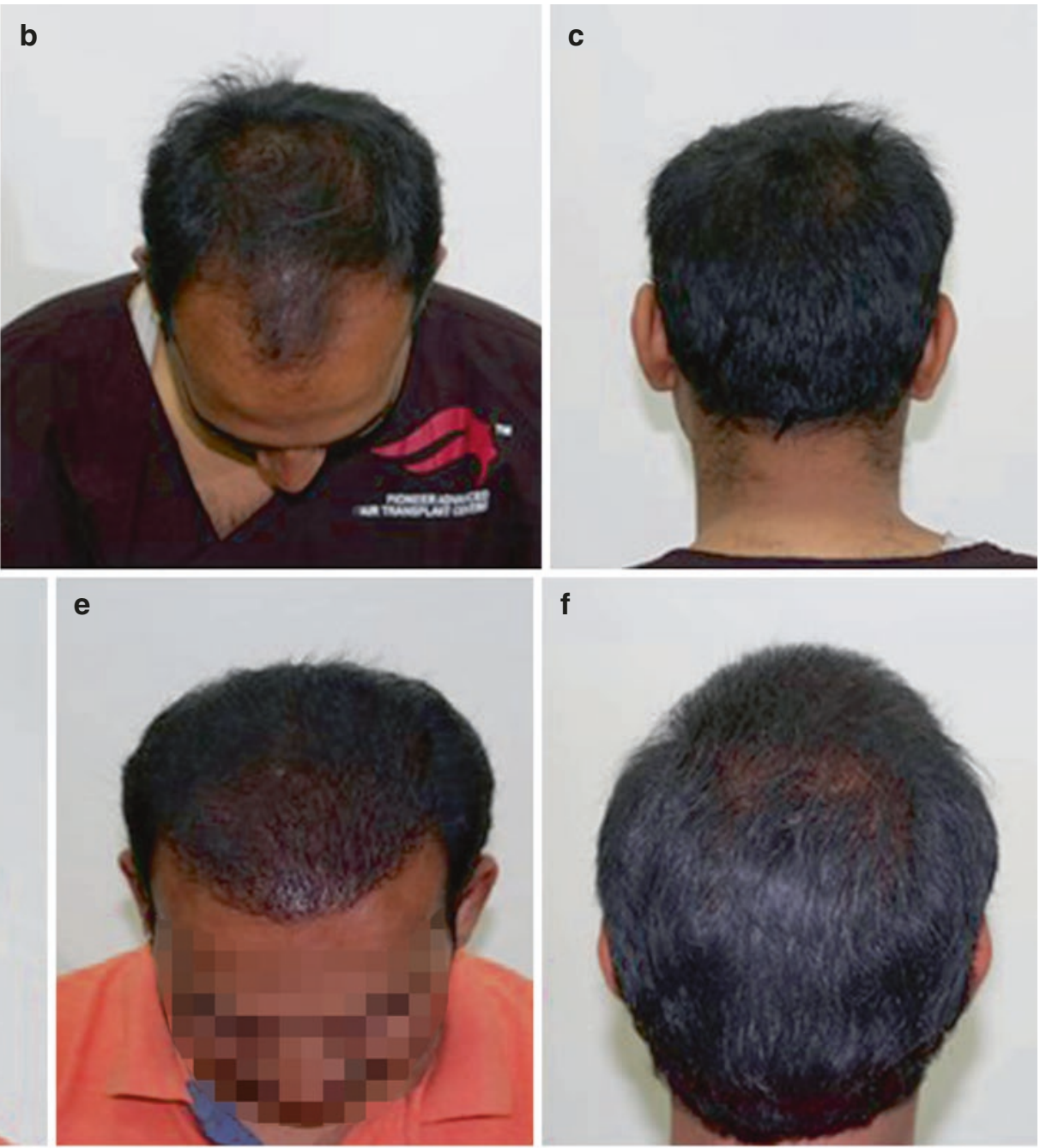

Fig. 34.26 (a-f) Case scenario 1 
Fig. 34.27 (a-d) Case scenario 2
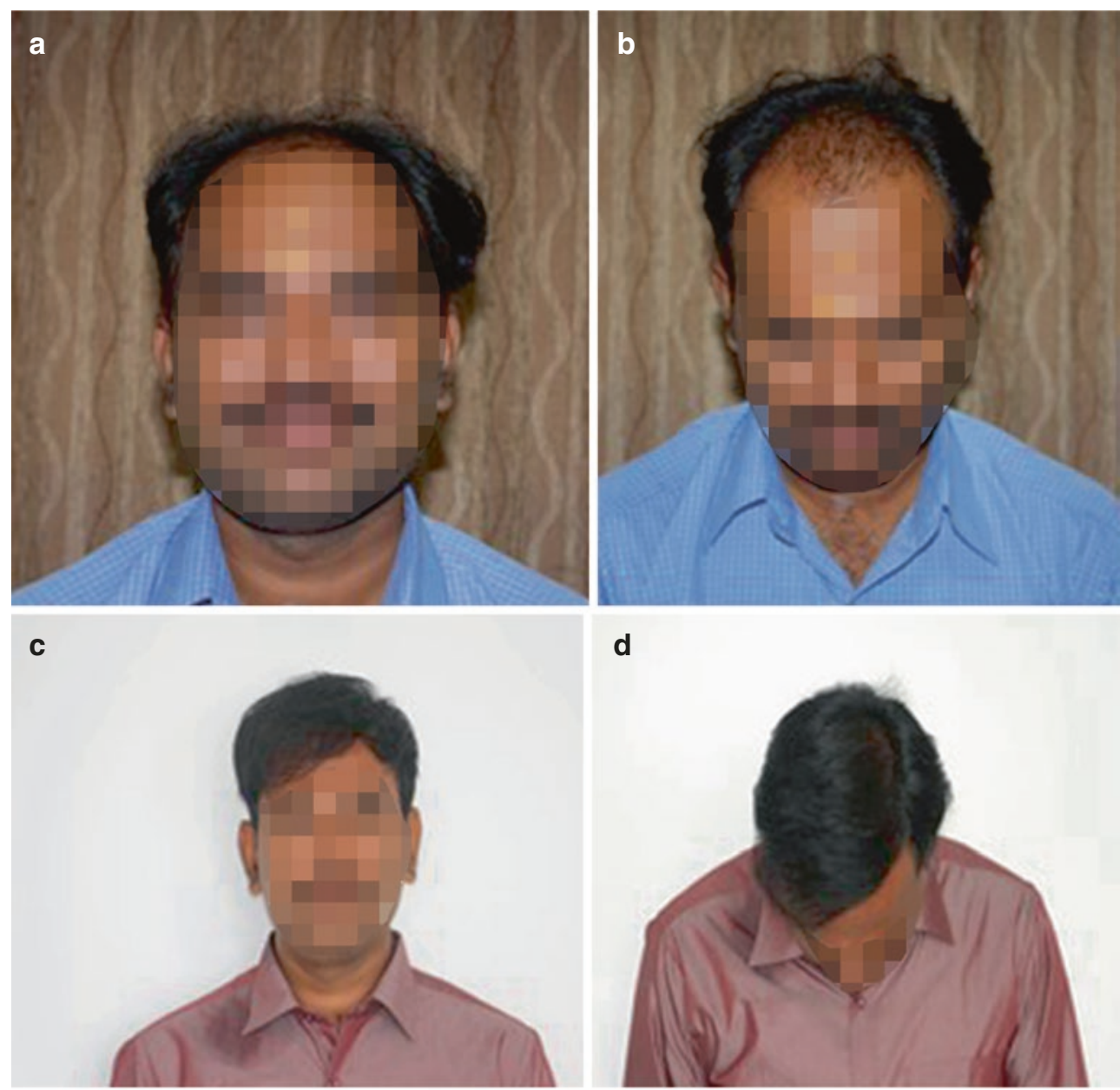

d

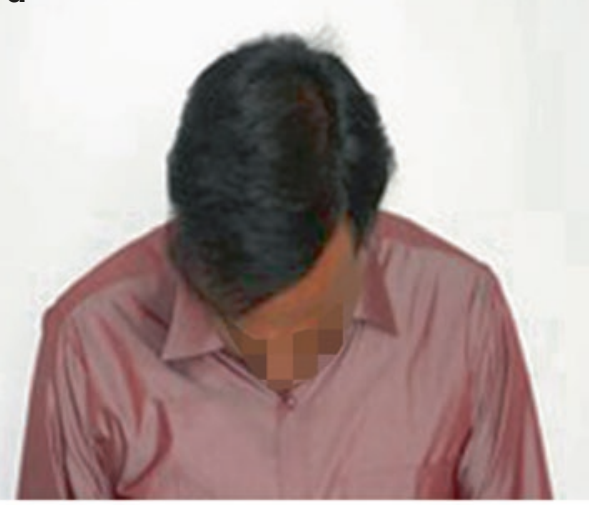

(CAssociation of Oral and Maxillofacial Surgeons of India

\section{References}

1. Orentreich N. Autografts in alopecias and other selected dermatological conditions. Ann N Y Acad Sci. 1959;83:463-79.

2. Beehner M. Nomenclature proposal for the zones and landmarks of the balding scalp. Dermatol Surg. 2001;27:375-80.

3. Bernstein R, Rassman WR, Seager D, et al. Standardizing the classification and description of follicular unit transplantation and mini-micrografting techniques. Dermatol Surg. 1998;24:957-63.

4. Haber RS. Pharmacologic management of pattern hair loss. Facial Plast Surg Clin North Am. 2004;12:181-9.

5. Price VH. Treatment of hair loss. N Engl J Med. 1999;341:964-73.

6. Unger W, Shapiro R. Hair transplantation. New York: Marcel Dekker; 2004. p. 49-56.
7. Bernstein RM, Rassman WR. Follicular transplantation: patient evaluation and surgical planning. Dermatol Surg. 1997;23:771-84.

8. Bernstein RM, Rassman WR. The logic of follicular unit transplantation. Dermatol Surg. 1999;17:277-95.

9. Rassman WR, Bernstein RM. Follicular unit extraction-minimally invasive surgery for hair transplantation. Dermatol Surg. 2002;28:720-8.

10. Shapiro R. Principles and techniques used to create a natural hairline in surgical hair restoration. Facial Plast Surg Clin North Am. 2004;12:201-17.

11. Cooley JE. Complications of hair transplantation. In: Unger WP, Shapiro R, editors. Hair transplantation. 4th ed. New York: Marcel Dekker; 2004. p. 568-73.

12. Bernstein RM, Rassman WR, Rashid N, Shiell RC. The art of repair in surgical hair restoration. Part 1: basic repair strategies. Dermatol Surg. 2002;28:738-94.

Open Access This chapter is licensed under the terms of the Creative Commons Attribution 4.0 International License (http://creativecommons. org/licenses/by/4.0/), which permits use, sharing, adaptation, distribution and reproduction in any medium or format, as long as you give appropriate credit to the original author(s) and the source, provide a link to the Creative Commons license and indicate if changes were made.

The images or other third party material in this chapter are included in the chapter's Creative Commons license, unless indicated otherwise in a credit line to the material. If material is not included in the chapter's Creative Commons license and your intended use is not permitted by statutory regulation or exceeds the permitted use, you will need to obtain permission directly from the copyright holder. 